\title{
THREE-DIMENSIONAL PRESENTATIONS FOR THE GROUPS OF ORDER AT MOST 30
}

\author{
GRAHAM ELLIS AND IRINA KHOLODNA
}

\begin{abstract}
For each group $G$ of order up to 30 we compute a small 3-dimensional $\mathrm{CW}$-space $X$ with $\pi_{1} X \cong G$ and $\pi_{2} X=0$, and we quantify the 'efficiency' of $X$. Furthermore, we give a theoretical result for treating the case when $G$ is a semi-direct product of two groups for which 3 -presentations are known. We also describe the $\mathbf{Z} G$-module structure on the second homotopy group $\pi_{2} X^{2}$ of the 2-skeleton of $X$. This module structure can in principle be used to determine the cohomology groups $H^{2}(G, A)$ and $H^{3}(G, A)$ with coefficients in a $\mathbf{Z} G$-module $A$. Our computations, which involve the Todd-Coxeter procedure for coset enumeration and the LLL algorithm for finding bases of integer lattices, are rather naive in that the LLL algorithm is applied to matrices of dimension a multiple of $|G|$. Thus, in their present form, our techniques can be used only on small groups (say of order up to several hundred). They can in principle be used to construct (crossed) $\mathbf{Z} G$-resolutions of $\mathbf{Z}$, but again, only for small $G$. The paper is accompanied by two attachment files. The first of these is a summary of our computations in HTML format. The second contains various GAP programs used in the computations.
\end{abstract}

\section{Introduction}

Recall that a presentation of a group $G$ consists of a set $\underline{x}$ that generates $G$, together with a set $\underline{r}$ of relations between these generators, such that every relation satisfied by the generators can be derived from $\underline{r}$. The notion is made precise by introducing the free group $F$ on $\underline{x}$, and taking $\underline{r}$ to be any subset of $F$ whose normal closure $R$ satisfies $F / R \cong G$. The elements of $\underline{r}$ are called relators when one wishes to emphasize that they are elements of the group $F$. For example, $<x, y \mid x^{2}, y^{3},(x y)^{2}>$ is a presentation of the symmetric group $S_{3}$ involving three relators $a:=x^{2}, b:=y^{3}, c:=(x y)^{2}$.

A 3-dimensional presentation (or more simply 3-presentation) of $G$ consists of the data $<\underline{x} \mid \underline{r}>$ together with a set $\underline{s}$ of 'identities between the relators', such that every identity satisfied by the relators can be derived from $\underline{s}$. For example, the above presentation of $S_{3}$ can be extended to a 3-presentation by setting

$$
\underline{s}=\left\{\left({ }^{x} a\right) a^{-1}, \quad\left({ }^{y} b\right) b^{-1}, \quad\left({ }^{y^{2}} a^{-1}\right)\left({ }^{y^{2}} c\right)\left({ }^{y} a^{-1}\right)\left({ }^{x^{-1}} c\right) a^{-1} c\left({ }^{y^{2}} b^{-1}\right)\left(y^{y^{2} x^{-1}} b^{-1}\right)\right\},
$$

where ${ }^{x} a$ is intended to be read as a conjugate $x a x^{-1}$. Note that any word constructed from conjugates of relators represents an element in $F$, and that each of the words in $\underline{s}$ represents the identity element. 
To make the notion of a 3-presentation more precise we use the 2-dimensional CW-space $K=K(\underline{x}, \underline{r})$ associated to the presentation $<\underline{x} \mid \underline{r}>$. This space has a single 0-cell, a 1-cell for each generator, and a 2-cell for each relator (attached in accordance with the generatorword spelled by the relator). There is an isomorphism $\pi_{1} K \cong G$, and the elements of $\pi_{2} K$ are defined to be the identities between relators. Note that there is a canonical group action $\pi_{1} K \times \pi_{2} K \rightarrow \pi_{2} K,(x, r) \mapsto{ }^{x} r$. The set $\underline{s}$ is taken to be any set of generators for the $\mathbf{Z} G$-module $\pi_{2} K$, with each generator exhibited as a word in conjugates of relators that represents the identity word in $F$. An alternative, purely algebraic, description of $\underline{s}$ is given below.

In this paper we explicitly construct a small 3-presentation $<\underline{x}|\underline{r}| \underline{s}>$ for each group $G$ of order less than 32. In each case we also determine the structure of the module of identities $\pi=\pi_{2} K(\underline{x}, \underline{r})$ by listing: (1) the rank $\operatorname{dim}_{\mathbf{Z}} \pi$ of the free $\mathbf{Z}$-module underlying $\pi$; (2) a minimal set of elements in $\oplus_{\underline{r}} \mathbf{Z} G$ that generate a $\mathbf{Z} G$-module isomorphic to $\pi$. Furthermore, for each group we list the integral homology $H_{n}(G, \mathbf{Z})$ in dimensions $n=1,2,3$ and relate this homology to the 'efficiency' of the given 3-presentation. Full details of computational results are presented in HTML format in Appendix A.

Our method requires the use of a computer algebra package such as GAP [21] or MAGMA [5]. In particular, it uses the Todd-Coxeter procedure for coset enumeration [12] and the LLL algorithm of Lenstra, Lenstra and Lovasz [27] for finding bases of integer lattices. The final section of the paper describes several GAP procedures that we have written to compute $\pi$ and $H_{3}(G, \mathbf{Z})$. These procedures are listed in Appendix B. Our procedure for $\mathrm{H}_{3}(G, \mathbf{Z})$ is generally not as efficient as existing methods, such as that described in [22]. (However, our procedure can be adapted to one for higher-dimensional integral homology groups $H_{n}(G, \mathbf{Z})$. We believe that for values of $n$ larger than about 5, and for small groups $G$, this might perform better than the method in [22].)

The motivation for the paper is two-fold.

(i) It is explained below how the module of identities $\pi$ is useful for computing the cohomology $H^{2}(G, A)$ and $H^{3}(G, A)$ of $G$ with coefficients in a $\mathbf{Z} G$-module $A$. The elements of the third cohomology group represent the homotopy 2-types $X$ with $\pi_{1} X=G$ and $\pi_{2} X=A$. An estimate for the number $\Lambda\left(2, p^{n}\right)$ of distinct homotopy 2-types with $|G| \times|A|=p^{n}, p$ a prime, is given in [17]. In a subsequent paper we intend to determine $\Lambda\left(2, p^{n}\right)$ precisely, for various low values of $p$ and $n$, by using the results obtained below to make explicit computations of third cohomology groups. (On a more conjectural level, we also hope at some future stage to combine the techniques of this paper with those given in [2] and [15] in order to determine the number $\Lambda\left(3, p^{n}\right)$ of distinct homotopy 3-types $X$ with $\left|\pi_{1} X\right| \times\left|\pi_{2} X\right| \times\left|\pi_{3} X\right|=p^{n}$.)

(ii) Several authors have developed sophisticated and powerful methods for computing identities among relators (and for tackling the related problem of computing free $\mathbf{Z} G$ resolutions). See for example the survey papers [4] and [7], and more recent papers such as [1], [10], [22] and [25]. Our second motivation was a desire to compare these methods with the relatively naive computer techniques described below. It turns out that for finite groups $G$ of small order (say up to order several hundred) our techniques would seem to be a useful alternative. For instance, existing techniques had suggested that one might need a minimum of four identities between relators to extend the standard presentation $<x, y \mid x^{2}, y^{3},(x y)^{2}>$ of $S_{3}$ to a 3-presentation; in Section 3 we illustrate our methods by verifying that the above set $\underline{s}$ of three identities suffices. 
Acknowledgements. The first author was based at the Max-Planck-Institut für Mathematik, Bonn, during the course of this work and would like to thank the institute for its generous hospitality. Both authors thank the Mathematics Department at the University of Wales, Bangor for its hospitality and useful discussions in August 1998. The second author is grateful to Forbairt for financial support.

\section{Further details on 3-presentations}

It might not be fully apparent from the above description of a 3-presentation why the elements of $\pi=\pi_{2} K(\underline{x}, \underline{r})$ represent 'identities' between the relators of the presentation $<\underline{x} \mid \underline{r}>$. To clarify the situation we now recall an algebraic description of $\pi$.

As before, let $F=F(\underline{x})$ be the free group on the set $\underline{x}$, and $R$ be the normal closure in $F$ of the set $\underline{r}$. Let $E$ denote the free group on the cartesian product $F \times \underline{r}$. The generators of $E$ are thus pairs $(f, r)$ with $f \in F, r \in \underline{r}$. There is a group action $F \times E \rightarrow E$ defined by $f^{\prime}(f, r)=\left(f^{\prime} f, r\right)$, and a homomorphism $\delta: E \rightarrow F$ defined by $\delta(f, r)=f r f^{-1}$. Elements in $E$ of the form

$$
v w v^{-1}\left({ }^{\delta(v)} w^{-1}\right)
$$

with $v, w \in E$ are called Peiffer commutators. Since any Peiffer commutator clearly lies in $\operatorname{ker} \delta$, we consider the quotient group

$$
C(\underline{r})=E / P
$$

where $P$ denotes the subgroup of $E$ that is normally generated by the set of all Peiffer commutators. The above action and homomorphism $\delta$ induce an action $F \times C(r) \rightarrow C(r)$, $(f, w) \mapsto{ }^{f} w$ and homomorphism $\partial: C(\underline{r}) \rightarrow F$. (As explained in [7], [30] and [2] the homomorphism $\partial$ has the structure of a free crossed module.)

The elements of $C(\underline{r})$ can be considered as formal expressions involving conjugates of relators, with those elements in ker $\partial$ representing 'identities' among relations. It is not difficult to show that the subgroup ker $\partial$ is abelian, and that the action of $F$ induces an action of $G$ on this subgroup, making ker $\partial$ into a $\mathbf{Z} G$-module. An isomorphism of $\mathbf{Z} G$-modules

$$
\pi_{2} K(\underline{x}, \underline{r}) \cong \operatorname{ker} \partial
$$

was proved by J. H. C. Whitehead [32]. This leads to the following purely algebraic definition.

Definition 1. A 3-presentation of a group $G$ consists of a presentation $\langle\underline{x}| \underline{r}>$ for $G$ together with a set $\underline{s}$ of elements in $C(\underline{r})$ that generate $\operatorname{ker}(\partial: C(\underline{r}) \rightarrow F(\underline{x}))$ as a $\mathbf{Z} G$ module.

In obtaining the above isomorphism Whitehead showed that there is a $G$-equivariant group isomorphism

$$
\pi_{2}\left(K^{2}, K^{1}\right) \cong C(\underline{r})
$$

where $K^{n}$ denotes the $n$-dimensional skeleton of the CW-space $K(\underline{x}, \underline{r})$. Note that specifying a set of elements of the relative homotopy group $\pi_{2}\left(K^{2}, K^{1}\right)$ that generates $\pi_{2}\left(K^{2}\right)$ is an extremely effective means of specifying how to attach 3-cells to $K^{2}$ in order to obtain a 3-dimensional CW-space $X$ with $\pi_{2} X=0, \pi_{1} X \cong G$. Thus a 3-presentation is equivalent to such a $\mathrm{CW}$-space $X$. 
There are fairly obvious strategies for finding presentations for finite groups specified by their multiplication table (such as permutation groups and matrix groups). Rather than discuss these here, we suppose that our finite group $G$ has been specified by means of a finite presentation $<\underline{x} \mid \underline{r}>$. Our method for computing $\underline{s}$ is based on the commutative diagram

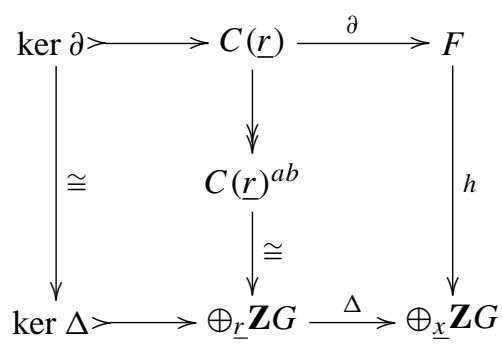

in which $\Delta$ is a homomorphism of $\mathbf{Z} G$-modules, and $h$ is a derivative (as defined in the following paragraph). Further details on this diagram are given in [7] and [30]. The important feature for us is that we can compute a suitable set $\underline{s}$ by first computing ker $\Delta$. In order to do this we recall a description of the homomorphism $\Delta$ given in terms of the WhiteheadReidemeister-Fox derivative.

Let $W$ be a $\mathbf{Z} G$-module, let $\phi: F \rightarrow G$ be the quotient homomorphism, and let an element $f \in F$ act on an element $w \in W$ by $f . w=\phi(f) w$. A function $\chi: F \rightarrow W$ is said to be a derivative if it satisfies the rule $\chi\left(f f^{\prime}\right)=\chi f+f \cdot \chi f^{\prime}$. Taking $x=\left\{x_{1}, \ldots, x_{m}\right\}$, it is readily seen that for each generator $x_{i}$ there is a unique derivative $\frac{\partial}{\partial x_{i}}: F \rightarrow \mathbf{Z} G$ that satisfies $\frac{\partial}{\partial x_{i}}\left(x_{i}\right)=1$ and $\frac{\partial}{\partial x_{i}}\left(x_{j}\right)=0$ for $i \neq j$.

Let us denote by $e_{i}^{1}$ the basis element of $\oplus_{x} \mathbf{Z} G$ corresponding to $x_{i} \in \underline{x}$. Similarly, we take $\underline{r}=\left\{a_{1}, \ldots, a_{n}\right\}$ and let $e_{i}^{2}$ denote the basis element of $\oplus_{\underline{r}} \mathbf{Z} G$ corresponding to $a_{i} \in \underline{r}$. The $\mathbf{Z} G$-module homomorphism $\Delta: \bigoplus_{\underline{r}} \mathbf{Z} G \rightarrow \bigoplus_{\underline{x}} \mathbf{Z} G$ is defined on basis elements by

$$
\Delta\left(e_{i}^{2}\right)=\sum_{j=1}^{m}\left(\frac{\partial a_{i}}{\partial x_{j}}\right) e_{j}^{1} .
$$

More details can be found in [7] and [30].

We now illustrate how these definitions and results are used to construct 3-presentations.

\section{Calculating a 3-presentation for $\mathbf{S}_{\mathbf{3}}$}

We start with the presentation $<x, y \mid x^{2}, y^{3},(x y)^{2}>$ for $G=S_{3}$, and as above set $a:=x^{2}, b:=y^{3}, c:=(x y)^{2}$. Then

$$
\begin{array}{lll}
\frac{\partial a}{\partial x}=1+x, & \frac{\partial b}{\partial x}=0, & \frac{\partial c}{\partial x}=1+x y, \\
\frac{\partial a}{\partial y}=0, & \frac{\partial b}{\partial y}=1+y+y^{2}, & \frac{\partial c}{\partial y}=x+y^{2} .
\end{array}
$$

The $\mathbf{Z} G$-modules $\oplus_{x} \mathbf{Z} G$ and $\oplus_{r} \mathbf{Z} G$ can be considered as free abelian groups of dimensions 12 and 18. In order to specify $\mathbf{Z}$-bases for these abelian groups we order the elements of $G, \underline{x}$ and $\underline{r}$ as follows: 


$$
\begin{aligned}
& G=\left\{g_{1}, \ldots, g_{6}\right\}=\left\{1, x, y, y^{2}, x y, x y^{2}\right\}, \\
& \underline{x}=\left\{x_{1}, x_{2}\right\}=\{x, y\}, \\
& \underline{r}=\left\{a_{1}, a_{2}, a_{3}\right\}=\{a, b, c\} .
\end{aligned}
$$

We identify the basis element $g_{i} e_{j}^{1}$ of $\oplus_{\underline{x}} \mathbf{Z} G$ with the standard basis element $(0, \ldots, 0$, $1,0, \ldots, 0)$ of $\mathbf{Z}^{12}$ whose $\{6(j-1)+i\}$ th coordinate is equal to 1 . Similarly we identify $g_{i} e_{j}^{2}$ with the standard basis element of $\mathbf{Z}^{18}$ whose $\{6(j-1)+i\}$ th coordinate is equal to 1. With respect to these $\mathbf{Z}$-bases the matrix of the homomorphism $\Delta: \mathbf{Z}^{18} \rightarrow \mathbf{Z}^{12}$ is:

$$
A=\left(\begin{array}{llllllllllllllllll}
1 & 1 & 0 & 0 & 0 & 0 & 0 & 0 & 0 & 0 & 0 & 0 & 1 & 0 & 0 & 0 & 1 & 0 \\
1 & 1 & 0 & 0 & 0 & 0 & 0 & 0 & 0 & 0 & 0 & 0 & 0 & 1 & 1 & 0 & 0 & 0 \\
0 & 0 & 1 & 0 & 0 & 1 & 0 & 0 & 0 & 0 & 0 & 0 & 0 & 1 & 1 & 0 & 0 & 0 \\
0 & 0 & 0 & 1 & 1 & 0 & 0 & 0 & 0 & 0 & 0 & 0 & 0 & 0 & 0 & 1 & 0 & 1 \\
0 & 0 & 0 & 1 & 1 & 0 & 0 & 0 & 0 & 0 & 0 & 0 & 1 & 0 & 0 & 0 & 1 & 0 \\
0 & 0 & 1 & 0 & 0 & 1 & 0 & 0 & 0 & 0 & 0 & 0 & 0 & 0 & 0 & 1 & 0 & 1 \\
0 & 0 & 0 & 0 & 0 & 0 & 1 & 0 & 1 & 1 & 0 & 0 & 0 & 1 & 1 & 0 & 0 & 0 \\
0 & 0 & 0 & 0 & 0 & 0 & 0 & 1 & 0 & 0 & 1 & 1 & 1 & 0 & 0 & 0 & 1 & 0 \\
0 & 0 & 0 & 0 & 0 & 0 & 1 & 0 & 1 & 1 & 0 & 0 & 0 & 0 & 0 & 1 & 0 & 1 \\
0 & 0 & 0 & 0 & 0 & 0 & 1 & 0 & 1 & 1 & 0 & 0 & 1 & 0 & 0 & 0 & 1 & 0 \\
0 & 0 & 0 & 0 & 0 & 0 & 0 & 1 & 0 & 0 & 1 & 1 & 0 & 0 & 0 & 1 & 0 & 1 \\
0 & 0 & 0 & 0 & 0 & 0 & 0 & 1 & 0 & 0 & 1 & 1 & 0 & 1 & 1 & 0 & 0 & 0
\end{array}\right) .
$$

A $\mathbf{Z}$-basis for ker $\Delta$ can be found using the computer algebra package GAP [21]. One enters the matrix $A$ and applies the command

NS := LLLReducedBasis(TransposedMat(A), "linearcomb").relations; to obtain, by means of the LLL algorithm [27], the following $\mathbf{Z}$-basis for ker $\Delta$ :

$$
\begin{array}{llll}
v_{1}= & (-1,1,0,0,0,0, & 0,0,0,0,0,0, & 0,0,0,0,0,0), \\
v_{2}=(0,0,0,-1,1,0, & 0,0,0,0,0,0, & 0,0,0,0,0,0), \\
v_{3}=(0,0,-1,0,0,1, & 0,0,0,0,0,0, & 0,0,0,0,0,0), \\
v_{4}=(0,0,0,0,0,0, & -1,0,1,0,0,0, & 0,0,0,0,0,0), \\
v_{5}=(0,0,0,0,0,0, & -1,0,0,1,0,0, & 0,0,0,0,0,0), \\
v_{6}= & (0,0,0,0,0,0, & 0,-1,0,0,1,0, & 0,0,0,0,0,0), \\
v_{7}= & (0,0,0,0,0,0, & 0,-1,0,0,0,1, & 0,0,0,0,0,0), \\
v_{8}= & (0,0,0,0,0,0, & 0,0,0,0,0,0, & 0,-1,1,0,0,0), \\
v_{9}=(-1,0,-1,-1,0,0, & -1,-1,0,0,0,0, & 1,1,0,1,0,0), \\
v_{10}=(0,0,0,0,0,0, & 0,0,0,0,0,0, & -1,0,0,0,1,0), \\
v_{11}=(-1,0,-1,-1,0,0, & -1,-1,0,0,0,0, & 1,1,0,0,0,1) .
\end{array}
$$

The $\mathbf{Z}$-basis for ker $\Delta$ is reduced in the sense that the norms of the basis vectors are 'as small as possible' [27]. (As a homomorphism of free abelian groups $\Delta$ is rather special in that its cokernel is free abelian. It might be possible to incorporate this extra information into the LLL algorithm and thereby increase the speed of computing the $\mathbf{Z}$-basis for ker $\Delta$. Alternatively, it might be possible to incorporate this extra information into one of the two algorithms described in [11]. ) 
In order to find a minimal subset MIN of $\underline{v}=\left\{v_{1}, \ldots, v_{11}\right\}$ that generates ker $\Delta$ as a $\mathbf{Z} G$-module we apply the following procedure.

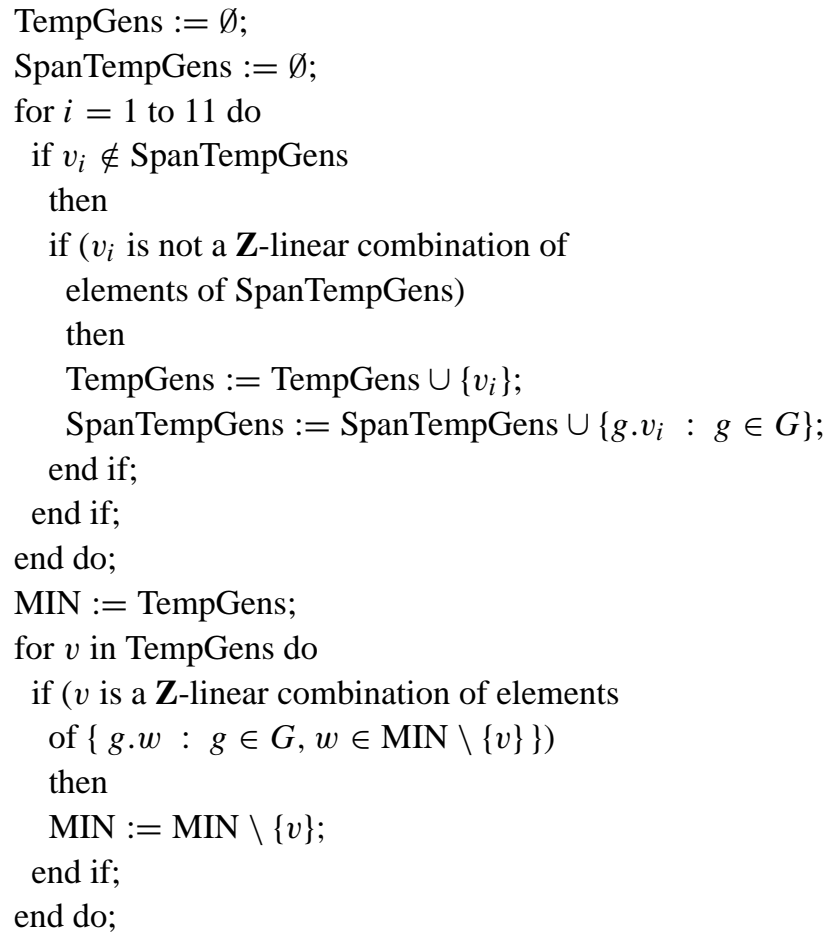

A time-consuming feature of the procedure is our somewhat unsophisticated method for testing whether a given vector $v$ is a $\mathbf{Z}$-linear combination of a given set of vectors $S$. Our method is to test whether the $\mathbf{Z}$-modules $L(S)$ and $L(S \cup\{v\})$ generated by $S$ and $S \cup\{v\}$ are identical. We first check easily computed properties, such as whether $\operatorname{rank}_{\mathbf{Q}}(S)=$ $\operatorname{rank}_{\mathbf{Q}}(S \cup\{v\})$. If these checks do not imply $L(S) \neq L(S \cup\{v\})$ then we use GAP's function HermiteNormalFormIntegerMat to check whether the Hermite Normal Forms of the sets $S$ and $S \cup\{v\}$ are identical; they are identical if and only if $L(S)$ is equal to $L(S \cup\{v\})$. (We believe that Gröbner basis techniques might lead to a more efficient procedure for determining MIN.)

The above procedure shows that ker $\Delta$ is minimally generated as a $\mathbf{Z} G$-module by the following three elements:

$$
\begin{aligned}
& v_{1}=(-1+x) e_{1}^{2}, \\
& v_{4}=(-1+y) e_{2}^{2}, \\
& v_{9}=\left(-1-y-y^{2}\right) e_{1}^{2}+(-1-x) e_{2}^{2}+\left(1+x+y^{2}\right) e_{3}^{2} .
\end{aligned}
$$

To complete the construction of a 3-presentation for $S_{3}$ we must find a set $\underline{s}$ of three elements in $C(\underline{r})$ whose images in $\oplus_{\underline{r}} \mathbf{Z} G$ generate the same module as $\left\{v_{1}, v_{4}, v_{9}\right\}$. Consider the following elements in $C(\underline{r})$ :

$$
\begin{aligned}
& w_{1}=\left({ }^{x} a\right) a^{-1}, \\
& w_{2}=\left({ }^{y} b\right) b^{-1} \\
& w_{3}=\left({ }^{y^{2}} a^{-1}\right)\left({ }^{y^{2}} c\right)\left({ }^{y} a^{-1}\right)\left({ }^{x^{-1}} c\right) a^{-1} c\left(y^{y^{2}} b^{-1}\right)\left({ }^{y^{2} x^{-1}} b^{-1}\right) .
\end{aligned}
$$

It is easily verified that each $w_{i}$ lies in ker $\partial$ and that the isomorphism ker $\partial \cong \operatorname{ker} \Delta$ maps 


$$
\begin{aligned}
& w_{1} \mapsto v_{1}, \\
& w_{2} \mapsto v_{4}, \\
& w_{3} \mapsto \bar{v}_{9}:=v_{9}-(1+x+y) v_{4},
\end{aligned}
$$

where the sets $\left\{v_{1}, v_{4}, v_{9}\right\}$ and $\left\{v_{1}, v_{4}, \bar{v}_{9}\right\}$ have the same $\mathbf{Z} G$-span. Hence

$$
<x, y\left|x^{2}, y^{3},(x y)^{2}\right| w_{1}, w_{2}, w_{3}>
$$

is a 3-presentation of $S_{3}$.

(An alternative 3-presentation for $S_{3}$ was calculated in [10] using groupoid techniques. This alternative involves four identities between relators, and has the form

$$
\begin{gathered}
\left.<x, y\left|a:=x^{2}, b:=y^{3}, d:=(y x)^{2}\right| a^{-1}\left({ }^{x} a\right), b^{-1}\left({ }^{y^{-1}} b\right),\left({ }^{x} d^{-1}\right)^{y^{-1}} d\right), \\
\left({ }^{y} a^{-1}\right) d a^{-1}\left({ }^{x} b^{-1}\right)\left({ }^{y^{-1}} d\right)\left({ }^{y^{-1}} a^{-1}\right) b^{-1}\left({ }^{y} d\right)>.
\end{gathered}
$$

A 3-presentation for $S_{3}$ involving just two identities between relators can be found by applying the above method to the 2-presentation $<x, y \mid x^{2} y^{-3}, x y x^{-1} y^{2}>$. We leave this as an exercise for the reader!)

The above generator $w_{3}$ was not plucked from thin air. Rather, it was extracted from the Cayley graph of $S_{3}$

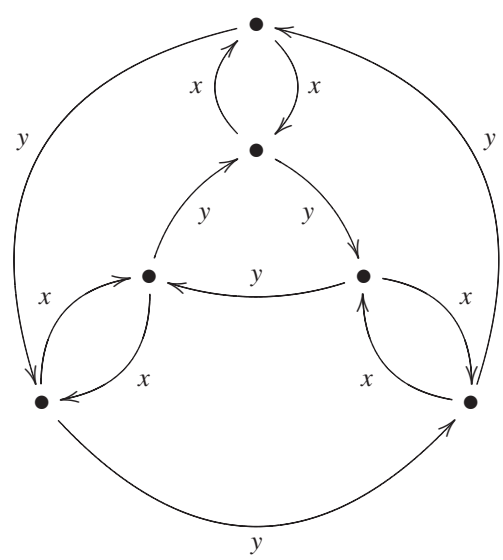

which can be viewed as representing a non-trivial identity involving three copies of $a$, two copies of $b$ and three copies of $c$. Our current method for finding a preimage in $C(\underline{r})$ of a general element in ker $\Delta$ involves human ingenuity and we are thus unable to automate this step of the calculation on a computer. (We should remark that the groupoid approach in [10] circumvents this step by performing computations directly in $C(\underline{r})$.)

Our computations can be seen as describing the low-dimensional terms of a free $\mathbf{Z G}$ resolution of $\mathbf{Z}$. More precisely, let $G$ now be an arbitrary group, let $\underline{r}_{3}$ denote the set of generators for the $\mathbf{Z} G$-module ker $\Delta$, and consider the module homomorphism $\Delta_{3}: \oplus_{\underline{r}_{3}} \mathbf{Z} G \rightarrow$ $\oplus_{\underline{r}} \mathbf{Z} G$ determined by sending a free $\mathbf{Z} G$-basis element to the corresponding element in $\operatorname{ker} \Delta$. We then have an exact sequence

$$
\oplus_{\underline{r}_{3}} \mathbf{Z} G \stackrel{\Delta_{3}}{\longrightarrow} \oplus_{\underline{r}} \mathbf{Z} G \stackrel{\Delta}{\longrightarrow} \oplus_{\underline{x}} \mathbf{Z} G \stackrel{\Delta_{1}}{\longrightarrow} \mathbf{Z} G \longrightarrow \mathbf{Z} \longrightarrow 0
$$

of $\mathbf{Z} G$-modules (where $\Delta_{1}$ sends the $\mathbf{Z} G$-basis element $z \in \underline{x}$ to the group-ring element $\phi(z)-1)$. Further terms in the resolution can be constructed inductively by using the above techniques to compute a minimal set $\underline{r}_{n+1}$ of generators for $\operatorname{ker} \Delta_{n}$. 
The size of $\underline{r}_{n}$ grows polynomially in $n$. Nevertheless, for certain small groups $G$ this method could be used to construct quite a number of terms in a resolution. Consider for instance the particularly favourable case when $G=D_{k}$ is the dihedral group of order $2 k$. For this group it is possible to construct a resolution with $\left|\underline{r}_{n}\right|=n+1$. Under the (possibly inaccurate) assumption that the above techniques yield this same resolution, the matrix of $\Delta_{n}$ would have $2 k n$ rows and $2 k(n+1)$ columns. The computer timings provided in the following section suggest that, for the dihedral group $G=D_{6}$ of order 12 , the above techniques could be used to produce the first 100 terms or so of a $\mathbf{Z} D_{6}$-resolution. This estimate compares favourably with estimates for the construction of a $\mathbf{Z} D_{6}$-resolution given in [22]. (However, it should be mentioned that the techniques in [22] also apply to infinite groups. Moreover, for large finite groups and low values of $n$, the techniques in [22] are more efficient.)

\section{Improving computational feasibility}

Consider a finite presentation $<\underline{x} \mid \underline{r}>$ of an arbitrary finite group $G$. The above method for obtaining a 3-presentation involves calculating the nullspace of an integer matrix $A$ with $|\underline{x}||G|$ rows and $|\underline{r}||G|$ columns. Table 1 lists the CPU time taken by the LLL algorithm in computing the nullspace of a fairly typical $n \times n$ matrix for various values of $n$. (The computation was performed using MAGMA [5] on a Sun Microsystems Ultra 10, and for each $n$ the matrix was taken to be the $n \times n$ identity matrix! The corresponding timings using GAP were slightly slower.)

Table 1: LLL algorithm timings

\begin{tabular}{rr}
\hline Value of $n$ & CPU time (seconds) \\
\hline 64 & 0.009 \\
128 & 0.060 \\
256 & 0.259 \\
512 & 1.660 \\
1024 & 14.250 \\
2048 & 115.999 \\
\hline
\end{tabular}

Thus, for groups $G$ of even quite modest order, the dimensions of the matrix $A$ pose a problem. We need techniques for breaking the computation of the nullspace of $A$ into a number of steps, the steps involving smaller matrices. One such technique is based on the following notion of 'morphism of presentations'.

Definition 2. By a morphism of presentations $<\underline{x}^{\prime}\left|\underline{r}^{\prime}>\rightarrow<\underline{x}\right| \underline{r}>$ we mean a settheoretic function $\lambda: \underline{x}^{\prime} \rightarrow F(\underline{x})$ such that the induced homomorphism $F\left(\underline{x}^{\prime}\right) \rightarrow F(\underline{x})$ maps the elements of $\underline{r}^{\prime}$ to distinct elements in $\underline{r}$.

We let $\pi_{1}(\underline{x}, \underline{r})$ denote the group $G$, and $\pi_{2}(\underline{x}, \underline{r})$ denote the $\mathbf{Z} G$-module ker $\partial: C(\underline{r}) \rightarrow$ $F(\underline{x})$.

Proposition 1. (i) Any such morphism of presentations induces a natural group homomorphism $\lambda_{1}: \pi_{1}\left(\underline{x}^{\prime}, \underline{r}^{\prime}\right) \rightarrow \pi_{1}(\underline{x}, \underline{r})$ and natural module homomorphism $\lambda_{2}: \pi_{2}\left(\underline{x}^{\prime}, \underline{r}^{\prime}\right) \rightarrow$ $\pi_{2}(\underline{x}, \underline{r})$. 
(ii) Considered as a morphism of free abelian groups, $\lambda_{2}$ maps the free abelian group $\pi_{2}\left(\underline{x}^{\prime}, \underline{r}^{\prime}\right)$ isomorphically onto a direct summand of the abelian group $\pi_{2}(\underline{x}, \underline{r})$.

(iii) If $\lambda_{1}$ is injective then assertion (ii) can be strengthened to an isomorphism of $\mathbf{Z H}$ modules

$$
\pi_{2}(\underline{x}, \underline{r}) \cong \pi_{2}\left(\underline{x}^{\prime}, \underline{r}^{\prime}\right) \oplus \mathbf{z} H P
$$

where $H=\pi_{1}\left(\underline{x}^{\prime}, \underline{r}^{\prime}\right), P$ is a projective $\mathbf{Z} H$-module, and $\oplus \mathbf{z} H$ denotes a direct sum of $\mathbf{Z} H$-modules.

Proof. We use the language of crossed modules [7] [30] [2]. The morphism of presentations clearly induces a morphism $\left\{C\left(\underline{r}^{\prime}\right) \rightarrow F\left(\underline{x}^{\prime}\right)\right\} \rightarrow\{C(\underline{r}) \rightarrow F(\underline{x})\}$ of free crossed modules, which in turn induces the homomorphisms $\lambda_{1}$ and $\lambda_{2}$.

Set $K=K\left(\underline{x}^{\prime}, \underline{r}^{\prime}\right)$ and $L=K(\underline{x}, \underline{r})$. The morphism of free crossed modules also induces a map $f: K \rightarrow L$ of 2-dimensional CW-spaces. This map can be converted into an inclusion $K \hookrightarrow L^{\prime}$ with $L^{\prime}$ a 2-dimensional CW-space that is homotopy equivalent to $L$. (The space $L^{\prime}$ is obtained by adding 2-cells to the mapping cylinder of the map of 1-skeleta $f: K^{1} \rightarrow L^{1}$.) By a result of M. Dyer [13] the boundary homomorphism $\partial: \pi_{2}\left(L^{\prime}, K\right) \rightarrow$ $\pi_{1}(K)$ is a projective crossed $\pi_{1}(K)$-module. Thus, by a result of J.G. Ratcliffe [28] [19], the abelianised group $\pi_{2}\left(L^{\prime}, K\right)^{a b}$ is a projective $\mathbf{Z} \pi_{1}(K)$-module. Thus, considered as an abelian group, $H_{2}\left(\tilde{L}^{\prime}, \tilde{K}\right) \cong \pi_{2}\left(L^{\prime}, K\right)^{a b}$ is free abelian. (Here, $\tilde{L}^{\prime}$ denotes the universal cover.) The exact homology sequence

$$
0=H_{3}\left(\tilde{L}^{\prime}, \tilde{K}\right) \rightarrow H_{2}(\tilde{K}) \rightarrow H_{2}(\tilde{L}) \rightarrow H_{2}\left(\tilde{L}^{\prime}, \tilde{K}\right) \rightarrow \operatorname{ker}\left(\lambda_{1}\right) \rightarrow 0
$$

implies that $\pi_{2}(K) \cong H_{2}(\tilde{K})$ is a direct summand of the free abelian group $\pi_{2}(L) \cong H_{2}(\tilde{L})$; to see this note that the image of $H_{2}(\tilde{L})$ in $H_{2}\left(\tilde{L}^{\prime}, \tilde{K}\right)$ is free abelian since it is a subgroup of a free abelian group. If $\operatorname{ker}\left(\lambda_{1}\right)=1$ then this exact sequence implies assertion (iii) with $P=\pi_{2}\left(L^{\prime}, K\right)^{a b}$.

To see how this proposition could have been used in the calculation of a 3-presentation for $S_{3}$, consider the following morphisms of presentations:

$$
\begin{aligned}
& <z\left|z^{2}>\rightarrow<x, y\right| x^{2}, y^{3},(x y)^{2}>, \quad z \mapsto x, \\
& <z\left|z^{2}>\rightarrow<x, y\right| x^{2}, y^{3},(x y)^{2}>, \quad z \mapsto x y, \\
& <u\left|u^{3}>\rightarrow<x, y\right| x^{2}, y^{3},(x y)^{2}>, \quad u \mapsto y .
\end{aligned}
$$

The module of identities $\pi_{2}\left(\{z\},\left\{z^{2}\right\}\right)$ is isomorphic to the submodule of $\mathbf{Z} C_{2}$ generated by $z-1$. This generator gets mapped by the first two morphisms to the elements $v_{1}=(x-1) e_{1}^{2}$ and $v_{10}=(x y-1) e_{3}^{2}$ in ker $\Delta: \oplus_{\underline{r}} \mathbf{Z} G \rightarrow \oplus_{\underline{x}} \mathbf{Z} G$, where $\Delta$ is as in Section 3 . The generator $1-u$ for the module of identities $\bar{\pi}_{2}\left(\{u\},\left\{u^{3}\right\}\right)$ gets mapped by the last morphism to the element $v_{4}=(y-1) e_{2}^{2}$. Proposition 1(ii) implies that the set $\underline{v}:=\left\{v_{1}, v_{4}, v_{10}\right\}$ can be extended to a $\mathbf{Z}$-basis of $\oplus_{\underline{r}} \mathbf{Z} G$. Moreover, the set $\underline{v}_{G}=\left\{v_{1}, v_{2}, v_{3}, v_{4}, v_{5}, v_{6}, v_{7}, v_{8}, v_{10}, v_{11}-v_{9}\right\}$, which is a basis for the abelian group $V$ spanned by $G . \underline{v}:=\left\{g . v_{1}, g . v_{4}, g . v_{10}: g \in S_{3}\right\}$, can be extended to a $\mathbf{Z}$-basis of $\oplus_{\underline{r}} \mathbf{Z} G$. (The computation of this extended basis can in fact be done by row operations over the rationals.) Let $\underline{u}:=\left\{u_{1}, \ldots, u_{9}\right\}$ be a $\mathbf{Z}$-basis for the complement of $V$, let $U$ denote this complement, and consider the restricted homomorphism $\Delta^{\prime}: U \rightarrow \oplus_{x} \mathbf{Z} G$. Then $\Delta^{\prime}$ is represented by a $12 \times 9$ integer matrix. We can determine a $\mathbf{Z}$-basis $\underline{b}$ for the nullspace of this $12 \times 9$ matrix, and take the union $\underline{b} \cup \underline{v}_{G}$ as a $\mathbf{Z}$-basis for the nullspace of ker $\Delta$. 


\section{Finitely generated abelian groups}

The above computer method is used in Section 7 to list 3-presentations for the nonabelian groups of order less than 32. The following proposition yields a 3-presentation for any finitely generated abelian group. This proposition is essentially given in [9] and [4]. It is also a particular case of Proposition 3 below.

Proposition 2. Suppose that $<\underline{x}|\underline{r}| \underline{s}>$ and $<\underline{x}^{\prime}\left|\underline{r}^{\prime}\right| \underline{s}^{\prime}>$ are 3-presentations for the groups $G$ and $G^{\prime}$. Then the direct product $G \times G^{\prime}$ has a 3-presentation of the form

$$
<\underline{x} \cup \underline{x}^{\prime}\left|\underline{r} \cup \underline{r}^{\prime} \cup \underline{x} \otimes \underline{x}^{\prime}\right| \underline{s} \cup \underline{s}^{\prime} \cup \underline{x} \otimes \underline{r}^{\prime} \cup \underline{x}^{\prime} \otimes \underline{r}>
$$

where the sets $\underline{x} \otimes \underline{x}^{\prime}, \underline{x} \otimes \underline{r}^{\prime}$ and $\underline{x}^{\prime} \otimes \underline{r}$ are defined as follows. For $x_{i} \in \underline{x}, x_{j}^{\prime} \in \underline{x}^{\prime}$ let $c_{i j}$ denote the commutator $x_{i} x_{j}^{\prime} x_{i}^{-1} x_{j}^{\prime-1}$ in the free group $F\left(\underline{x} \cup \underline{x}^{\prime}\right)$ generated by $\underline{x} \cup \underline{x}^{\prime}$. Then

$$
\underline{x} \otimes \underline{x}^{\prime}=\left\{c_{i j}: x_{i} \in \underline{x}, x_{j}^{\prime} \in \underline{x}^{\prime}\right\} .
$$

For $x_{i} \in \underline{x}, x_{j}^{\prime} \in \underline{x}^{\prime}, r=x_{i_{1}}^{\epsilon_{1}} x_{i_{2}}^{\epsilon_{2}} \ldots x_{i_{n}}^{\epsilon_{n}}, r^{\prime}=x_{j_{1}}^{\prime \epsilon_{1}} x_{j_{2}}^{\prime \epsilon_{2}} \ldots x_{j_{n}}^{\prime \epsilon_{n}}$ with $\epsilon_{i}=1$ or -1 , we set

$$
\begin{gathered}
c\left(x_{i}, x_{j}^{\prime}\right)=c_{i j}, \\
c\left(x_{i}, x_{j}^{\prime-1}\right)={ }^{x_{j}^{\prime-1}} c_{i j}^{-1}, \\
c\left(x_{i}^{-1}, x_{j}^{\prime}\right)={ }^{x_{i}^{-1}} c_{i j}^{-1},
\end{gathered}
$$$$
c\left(x_{i}, r^{\prime}\right)=c\left(x_{i}, x_{j_{1}}^{\prime \epsilon_{1}}\right)^{x_{j_{1}}^{\prime \epsilon}} c\left(x_{i}, x_{j_{2}}^{\prime \epsilon_{2}}\right)^{x_{j_{1}}^{\prime \epsilon_{1}} x_{j_{2}}^{\prime \epsilon_{2}}} c\left(x_{i}, x_{j_{3}}^{\prime \epsilon_{3}}\right) \ldots x_{j_{1}}^{\epsilon_{1}} x_{j_{2}}^{\prime \epsilon_{2}} \ldots x_{j_{n-1}}^{\prime \epsilon_{n-1}} c\left(x_{i}, x_{j_{n}}^{\prime \epsilon_{n}}\right),
$$$$
c\left(r, x_{j}^{\prime}\right)={ }^{x_{1}} x_{i_{2}}^{\epsilon_{2}} \ldots x_{i_{n-1}}^{\epsilon_{n-1}} c\left(x_{i_{n}}^{\epsilon_{n}}, x_{j}^{\prime}\right) \ldots x_{i_{1}}^{\epsilon_{1}} x_{i_{2}}^{\epsilon_{2}} c\left(x_{i_{3}}^{\epsilon_{2}}, x_{j}^{\prime}\right){ }^{x_{1_{1}}^{\epsilon_{1}}} c\left(x_{i_{2}}^{\epsilon_{2}}, x_{j}^{\prime}\right) c\left(x_{i_{1}}^{\epsilon_{1}}, x_{j}^{\prime}\right) .
$$

Then

$$
\begin{gathered}
\underline{x} \otimes \underline{r}^{\prime}=\left\{{ }^{x} r^{\prime} r^{\prime-1} c\left(x, r^{\prime}\right)^{-1}: x \in \underline{x}, r^{\prime} \in \underline{r}^{\prime}\right\}, \\
\underline{x}^{\prime} \otimes \underline{r}=\left\{{ }^{x^{\prime}} r r^{-1} c\left(r, x^{\prime}\right): x^{\prime} \in \underline{x^{\prime}}, r \in \underline{r}\right\} .
\end{gathered}
$$

Proof. We use the language of crossed complexes [7] [30] [2]. The 3-presentation < $\underline{x}|\underline{r}| \underline{s}>$ gives rise to a 3 -dimensional CW-space $X$ with $\pi_{1} X \cong G, \pi_{2} X=0$ and associated free crossed complex

$$
C(X)_{*}: \quad \oplus_{\underline{s}} \mathbf{Z} G \rightarrow C(\underline{r}) \stackrel{\partial}{\rightarrow} F(\underline{x}) .
$$

We say that the 3-presentation generates $C(X)_{*}$. Similarly the triple $<\underline{x}^{\prime}\left|\underline{r}^{\prime}\right| \underline{s}^{\prime}>$ gives rise to a space $X^{\prime}$ and generates a free crossed complex $C\left(X^{\prime}\right)_{*}$. The product space $X \times X^{\prime}$ has $\pi_{1}\left(X \times X^{\prime}\right) \cong G \times G^{\prime}$ and $\pi_{2}\left(X \times X^{\prime}\right)=0$. Any generating triple for $C\left(X \times X^{\prime}\right)_{*}$ will be a 3-presentation for $G \times G^{\prime}$. In fact, one can read off the 3-presentation of the proposition from the description of the free crossed complex $C\left(X \times X^{\prime}\right)$ given in [6] (see also [2, page 126]). (Note that the function $c\left(u, u^{\prime}\right)$ is defined inductively by the commutator relations $\left.c\left(x, u^{\prime} v^{\prime}\right)=c\left(x, u^{\prime}\right){ }^{u^{\prime}} c(x, v), c\left(u v, x^{\prime}\right)={ }^{u} c\left(v, x^{\prime}\right) c\left(u, x^{\prime}\right).\right)$

We illustrate this proposition by using it to construct a 3-presentation of the abelian group $C_{2} \times C_{4}$. The method of the preceding sections yields 3 -presentations

$$
<x\left|a:=x^{2}\right|\left({ }^{x} a\right) a^{-1}>, \quad<y\left|b:=y^{4}\right|\left({ }^{y} b\right) b^{-1}>
$$


of the cyclic groups $C_{2}$ and $C_{4}$. Applying the proposition to these 3-presentations yields the 3-presentation

$$
\begin{aligned}
<x, y\left|x^{2}, y^{4}, c:=x y x^{-1} y^{-1}\right| & \left({ }^{x} a\right) a^{-1},\left({ }^{y} a\right) a^{-1}\left({ }^{x} c\right) c, \\
& \left({ }^{y} b\right) b^{-1},\left({ }^{x} b\right) b^{-1}\left(y^{3} c^{-1}\right)\left({ }^{y^{2}} c^{-1}\right)\left({ }^{y} c^{-1}\right) c^{-1}>
\end{aligned}
$$

of $C_{2} \times C_{4}$.

The computer calculations of Section 7 show that, for an arbitrary direct product $G \times G^{\prime}$, Proposition 2 does not necessarily yield the smallest possible set $\underline{s}$ of generating identities between relators.

\section{Split extensions}

Suppose that $<\underline{x}|\underline{r}| \underline{s}>$ and $<\underline{x}^{\prime}\left|\underline{r}^{\prime}\right| \underline{s}^{\prime}>$ are 3-presentations for groups $G$ and $N$, and that $\alpha: G \rightarrow \operatorname{Aut}(N)$ is an action of $G$ on $N$. We would like to construct a 3-presentation for the semi-direct product $N \times_{\alpha} G$. (Recall that this semi-direct product has underlying set $N \times G$, and multiplication is defined by $(n, g)\left(n^{\prime}, g^{\prime}\right)=\left(n^{\alpha(g)} n^{\prime}, g g^{\prime}\right)$.)

Note that for each $g \in G$ we can construct a (non-unique) commutative diagram of group homomorphisms

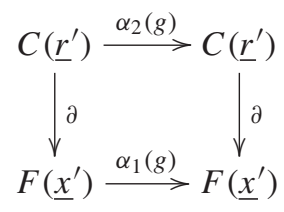

such that: $\alpha_{2}(g)$ preserves the action of $F\left(\underline{x}^{\prime}\right)$; the induced homomorphism on coker $\partial=N$ is equal to the automorphism $\alpha(g)$. (The homomorphisms $\alpha_{1}(g), \alpha_{2}(g)$ are not necessarily group automorphisms.) Suppose that one such diagram has been chosen for each $g \in G$.

For an element $x \in F(\underline{x})$ representing $g \in G$, and elements $f \in F\left(\underline{x}^{\prime}\right)$ and $w \in C\left(\underline{r}^{\prime}\right)$, we denote $\alpha_{1}(g)(f)$ by ${ }^{\alpha(\bar{x})} f$ and $\alpha_{2}(g)(w)$ by ${ }^{\alpha(x)} w$.

Proposition 3. Suppose that $<\underline{x}|\underline{r}| \underline{s}>$ and $<\underline{x}^{\prime}\left|\underline{r}^{\prime}\right| \underline{s}^{\prime}>$ are 3-presentations for the groups $G$ and $N$, and that $\alpha: G \rightarrow \operatorname{Aut}(N)$ is an action of $G$ on $N$. Then the semi-direct product $N \times{ }_{\alpha} G$ has a 3-presentation of the form

$$
<\underline{x} \cup \underline{x}^{\prime}\left|\underline{r} \cup \underline{r}^{\prime} \cup \underline{x} \otimes_{\alpha} \underline{x}^{\prime}\right| \underline{s} \cup \underline{s}^{\prime} \cup \underline{x} \otimes_{\alpha} \underline{r}^{\prime} \cup \underline{x}^{\prime} \otimes_{\alpha} \underline{r}>
$$

where the sets $\underline{x} \otimes_{\alpha} \underline{x}^{\prime}, \underline{x} \otimes_{\alpha} \underline{r}^{\prime}$ and $\underline{x}^{\prime} \otimes_{\alpha} \underline{r}$ are defined as follows. For $x_{i} \in \underline{x}, x_{j}^{\prime} \in \underline{x^{\prime}}$ let $c_{i j}$ denote the element $x_{i} x_{j}^{\prime} x_{i}^{-1}\left({ }^{\alpha\left(x_{i}\right)} x_{j}^{\prime}\right)^{-1}$ in the free group $F\left(\underline{x} \cup \underline{x}^{\prime}\right)$ generated by $\underline{x} \cup \underline{x^{\prime}}$. Then

$$
\begin{gathered}
\underline{x} \otimes_{\alpha} \underline{x}^{\prime}=\left\{c_{i j}: x_{i} \in \underline{x}, x_{j}^{\prime} \in \underline{x^{\prime}}\right\}, \\
\underline{x} \otimes_{\alpha} \underline{r}^{\prime}=\left\{{ }^{x} r^{\prime}\left({ }^{\alpha(x)} r^{\prime}\right)^{-1} c\left(x, r^{\prime}\right)^{-1}: x \in \underline{x}, r^{\prime} \in \underline{r}^{\prime}\right\}, \\
\underline{x}^{\prime} \otimes_{\alpha} \underline{r}=\left\{{ }^{x^{\prime}} r r^{-1} c\left(r, x^{\prime}\right): x^{\prime} \in \underline{x}^{\prime}, r \in \underline{r}\right\},
\end{gathered}
$$

where $c(-,-): F(\underline{x}) \times F\left(\underline{x}^{\prime}\right) \rightarrow C\left(\underline{r} \cup \underline{r}^{\prime} \cup \underline{x} \otimes_{\alpha} \underline{x}^{\prime}\right)$ is a function that satisfies

$$
c\left(x_{i}, x_{j}^{\prime}\right)=c_{i j},
$$




$$
\begin{gathered}
c\left(1, u^{\prime}\right)=c(u, 1)=1, \\
c\left(u, u^{\prime} v^{\prime}\right)=c\left(u, u^{\prime}\right){ }^{\left({ }^{(u)} u^{\prime}\right)} c\left(u, v^{\prime}\right), \\
c\left(u v, u^{\prime}\right)={ }^{u} c\left(v, u^{\prime}\right) c\left(u,{ }^{\alpha(v)} u^{\prime}\right) .
\end{gathered}
$$

Proof. It is an elementary result in the theory of group presentations [26] that $<\underline{x} \cup \underline{x}^{\prime} \mid \underline{r} \cup$ $\underline{r}^{\prime} \cup \underline{x} \otimes_{\alpha} \underline{x}^{\prime}>$ constitutes a presentation of the semi-direct product $N \times{ }_{\alpha} G$.

For convenience let us set $F:=F\left(\underline{x} \cup \underline{x}^{\prime}\right), M:=C\left(\underline{r} \cup \underline{r}^{\prime} \cup \underline{x} \otimes_{\alpha} \underline{x}^{\prime}\right)$ and consider the free crossed module $\partial: M \rightarrow F$. The properties of the function $c(-,-): F(\underline{x}) \times F\left(\underline{x}^{\prime}\right) \rightarrow$ $M$ stated in the proposition enable one to evaluate $c\left(u, u^{\prime}\right)$ as an element of $M$ for each $u \in F(\underline{x}), u^{\prime} \in F\left(\underline{x}^{\prime}\right)$. To show that this evaluation yields a unique element $c\left(u, u^{\prime}\right)$ of $M$ it suffices to establish, using the crossed module properties of $\partial$, that $c\left(u,\left(u_{1}^{\prime} u_{2}^{\prime}\right) u_{3}^{\prime}\right)=$ $c\left(u, u_{1}^{\prime}\left(u_{2}^{\prime} u_{3}^{\prime}\right)\right), c\left(\left(u_{1} u_{2}\right) u_{3}, u^{\prime}\right)=c\left(u_{1}\left(u_{2} u_{3}\right), u^{\prime}\right)$, and that $c\left(u_{1} u_{2}, u_{1}^{\prime} u_{2}^{\prime}\right)$ does not depend on which variable is expanded first.

Consider the commutative diagram

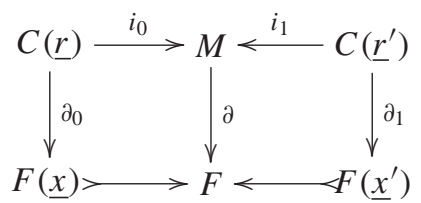

in which each vertical arrow is the canonical free crossed module, and the horizontal arrows are morphisms of crossed modules induced by set inclusions. Let $K$ be the $F$-subgroup of $M$ generated by the union of the sets

$$
i_{0}\left(\operatorname{ker} \partial_{0}\right) \cup i_{1}\left(\operatorname{ker} \partial_{1}\right)
$$

and

$$
\left\{{ }^{x} r^{\prime}\left({ }^{\alpha(x)} r^{\prime}\right)^{-1} c\left(x, r^{\prime}\right)^{-1}: x \in \underline{x}, r^{\prime} \in \underline{r}^{\prime}\right\} \cup\left\{{ }^{x^{\prime}} r r^{-1} c\left(r, x^{\prime}\right): x^{\prime} \in \underline{x^{\prime}}, r \in \underline{r}\right\} .
$$

It is routine to check that $K$ lies in the kernel of $\partial: M \rightarrow F$. We need to show that $K=\operatorname{ker} \partial$.

Consider the induced homomorphism $\bar{\partial}: M / K \rightarrow F$. Now im $\bar{\partial}$ is a subgroup of a free group, and hence free. We can thus choose a group-theoretic section $\eta$ : im $\bar{\partial} \rightarrow M / K$ to $\bar{\partial}$. (This section will not necessarily preserve the $F$-action.) Set $\sigma:=\eta \bar{\partial}: M / K \rightarrow M / K$. Let $S$ be a set of elements in $M / K$ that generates $M / K$ as a group. It is readily seen that $\operatorname{ker} \bar{\partial}$ is normally generated (as a group) by the elements $s \sigma(s)^{-1}$ for $s \in S$. Thus, to prove the proposition, it suffices to show that $\sigma(s)=s$ for all $s \in S$. We shall take

$$
S:=\left\{{ }^{f} r,{ }^{f} r^{\prime},{ }^{f} c\left(x, x^{\prime}\right): f \in F, x \in \underline{x}, x^{\prime} \in \underline{x^{\prime}}, r \in \underline{r}, r^{\prime} \in \underline{r}^{\prime}\right\}
$$

where, for convenience, we specify elements in $M / K$ by their preimage in $M$. Any element $f \in F$ can be written as a product of the form $f=(\bar{\partial} m) u u^{\prime}$ with $m \in M / K$, $u \in F(\underline{x}), u^{\prime} \in F\left(\underline{x}^{\prime}\right)$. For any element $w \in M / K$ the crossed module properties of $\bar{\partial}$ imply $\sigma\left({ }^{(\bar{\partial} m) u u^{\prime}} w\right)=m \sigma\left({ }^{u u^{\prime}} w\right) m^{-1}$. To prove the proposition it thus suffices to show that $\sigma\left({ }^{u u^{\prime}} r\right)=u u^{\prime} r, \sigma\left(u u^{\prime} r^{\prime}\right)=u^{\prime} r^{\prime}$, and $\sigma\left(u u^{\prime} c\left(x, x^{\prime}\right)\right)=u u^{\prime} c\left(x, x^{\prime}\right)$ for $u \in F(\underline{x})$, $u^{\prime} \in F\left(\underline{x}^{\prime}\right), x \in \underline{x}, x^{\prime} \in \underline{x}^{\prime}, r \in \underline{r}, r^{\prime} \in \underline{r}^{\prime}$.

Since $i_{0}\left(\operatorname{ker} \partial_{0}\right)$ and $i_{1}\left(\right.$ ker $\left.\partial_{1}\right)$ lie in $K$, we can assume

$$
\begin{gathered}
\sigma\left({ }^{u} t\right)={ }^{u} t, \\
\sigma\left({ }^{u^{\prime}} t^{\prime}\right)={ }^{u^{\prime}} t^{\prime}
\end{gathered}
$$


for $u \in F(\underline{x}), u^{\prime} \in F\left(\underline{x}^{\prime}\right), \quad t \in i_{0}(C(\underline{r})), t^{\prime} \in i_{1}\left(C\left(\underline{r}^{\prime}\right)\right)$.

Let us write

$$
<u, u^{\prime}>:=u u^{\prime} u^{-1 u} u^{\prime-1}
$$

The Reidemeister-Schreier rewriting process [26] yields a free generating set for im $\bar{\partial}$ which includes the elements $u^{\prime}<u, x^{\prime}>u^{\prime-1} t^{\prime}$ for all $u \in F(\underline{x}), u^{\prime} \in F\left(\underline{x}^{\prime}\right), x^{\prime} \in \underline{x}^{\prime}$, where $t^{\prime}$ is some element in $i_{1}\left(C\left(\underline{r}^{\prime}\right)\right)$ determined by $u, u^{\prime}, x^{\prime}$. Since $\partial\left({ }^{\prime} c\left(u, x^{\prime}\right)\right)=u^{\prime}<u, x^{\prime}>u^{\prime-1}$, we can choose $\eta$ such that

$$
\sigma\left({ }^{u^{\prime}} c\left(u, x^{\prime}\right)\right)={ }^{u^{\prime}} c\left(u, x^{\prime}\right)
$$

This equality can be extended to

$$
\sigma\left({ }^{u^{\prime}} c\left(u, v^{\prime}\right)\right)={ }^{u^{\prime}} c\left(u, v^{\prime}\right)
$$

for $v^{\prime} \in F\left(\underline{x}^{\prime}\right)$ by expressing $v^{\prime}$ in terms of generators and expanding. We also have

$$
\sigma\left({ }^{u} c\left(v, u^{\prime}\right)\right)=\sigma\left(c\left(u v, u^{\prime}\right) c\left(u,{ }^{\alpha v} u^{\prime}\right)^{-1}\right)={ }^{u} c\left(v, u^{\prime}\right)
$$

for $v \in F(\underline{x})$. It is now routine to verify that

$$
\sigma\left({ }^{u u^{\prime}} r\right)=\sigma\left({ }^{u} c\left(r, u^{\prime}\right)^{-1} u r\right)={ }^{u} c\left(r, u^{\prime}\right)^{-1} u r={ }^{u u^{\prime}} r .
$$

The verification of the equalities $\sigma\left({ }^{u u^{\prime}} r^{\prime}\right)={ }^{u u^{\prime}} r^{\prime}$ and $\sigma\left({ }^{u u^{\prime}} c\left(x, x^{\prime}\right)\right)={ }^{u u^{\prime}} c\left(x, x^{\prime}\right)$ is similar.

A version of Proposition 3 was first proved by Y.G. Baik in his PhD thesis (see [4, Theorem 3.2]) and extended by Baik, J. Harlander and S. Pride [1] to a more general result on the module of identities of general group extensions. Their results are expressed using the language of 'pictures over presentations'. Our proof of Proposition 3 uses different techniques from those employed in [4] and [1].

The computer calculations of Section 7 show that Proposition 3 does not necessarily yield the smallest possible set $\underline{s}$ of generating identities between relators. Furthermore, it should be noted that Proposition 3 cannot be used to extend an arbitrary presentation of a semi-direct product to a 3-presentation.

We illustrate Proposition 3 by using it to obtain a 3-presentation of the semi-direct product $C_{3} \times{ }_{\alpha} C_{2}$ where the generator $x$ of $C_{2}$ acts on the generator $y$ of $C_{3}$ by ${ }^{x} y=y^{-1}$. We have 3-presentations $<x\left|a:=x^{2}\right|\left({ }^{x} a\right) a^{-1}>$ and $<x\left|b:=y^{3}\right|\left({ }^{y} b\right) b^{-1}>$ of $C_{2}$ and $C_{3}$. Applying the proposition to these yields the 3-presentation

$$
\begin{gathered}
<x, y\left|x^{2}, y^{3}, c:=x y x^{-1} y\right| \quad\left({ }^{x} a\right) a^{-1},\left({ }^{x} b\right) b\left({ }^{y^{-2}} c^{-1}\right)\left({ }^{y^{-1}} c^{-1}\right) c^{-1}, \\
\left({ }^{y} b\right) b^{-1},\left({ }^{y} a\right) a^{-1}\left({ }^{x} c\right)\left({ }^{y} c^{-1}\right)>
\end{gathered}
$$

of $S_{3} \cong C_{3} \times{ }_{\alpha} C_{2}$. Note that this is a variant of the 3-presentation for $S_{3}$ given in Section 3 .

\section{The nonabelian groups of order at most 30}

We have used the above techniques to list, in an attached HTML file (see Appendix A), the following data for each of the 45 nonabelian groups $G$ of order at most 30 .

(i) The order $|G|$ and, where appropriate, name of $G$. 
(ii) A 3-presentation $<\underline{x}|\underline{r}| \underline{s}>$ for $G$. The set $\underline{s}$ is given using the convention that the first relator in $\underline{r}$ is denoted by $a$, the second relator is denoted by $b$, and so on. (We have followed [8] in our choice of generators, relators and names for the groups.)

(iii) A set $\underline{v}$ of elements in $\oplus_{r} \mathbf{Z} G$ that generate a $\mathbf{Z} G$-submodule that is isomorphic to the $\mathbf{Z} G$-module of identities $\pi=\pi_{2} K(\underline{x}, \underline{r})$. We use the convention that $\oplus_{r} \mathbf{Z} G$ has a basis $\left\{e_{a}, e_{b}, \ldots\right\}$ with $e_{a}$ corresponding to the first relator in $\underline{r}$, with $e_{b}$ corresponding to the second relator in $\underline{r}$, and so on.

(iv) The rank rk $\pi$ of the free abelian group underlying the module of identities $\pi=$ $\pi_{2} K(\underline{x}, \underline{r})$.

(v) The cardinality $|\underline{s}|$ of the set $\underline{s}$.

(vi) The integral homology group $H_{n}(G)=H_{n}(G, \mathbf{Z})$ for $n=1,2,3$. (In the following section these homology groups are related to the 'efficiency' of the given 3-presentation. Our method for calculating homology is explained in Section 9.)

A summary of the HTML file in Appendix A is given in Table 2.

This summary omits the details of the sets $\underline{s}$ and $\underline{v}$. For example, for the presentation $<x, y \mid x^{2}, y^{3},(x y)^{2}>$ of $S_{3}$ the HTML file lists the three elements of $\underline{s}$ as

$$
\left({ }^{x} a\right) a^{-1}, \quad\left({ }^{y} b\right) b^{-1}, \quad\left({ }^{y^{2}} a^{-1}\right)\left({ }^{y^{2}} c\right)\left({ }^{y} a^{-1}\right)\left({ }^{x^{-1}} c\right) a^{-1} c\left({ }^{y^{2}} b^{-1}\right)\left({ }^{y^{2} x^{-1}} b^{-1}\right)
$$

and the three elements of $v$ as

$$
(x-1) e_{a},(y-1) e_{b},\left(-1-y-y^{2}\right) e_{a}+(-1-x) e_{b}+\left(1+x+y^{2}\right) e_{c} .
$$

The dihedral groups $D_{n}$ of order $2 n$ have all been presented using two generators and three relators. However, for odd values of $n$, these groups also admit the presentation $<x, y \mid x^{2} y^{-n}, x y x^{-1} y^{(n-1)}>$. The above methods can be used to show that the module of identity of this two-relator presentation for odd $n$ is generated by just two elements (for small values of $n$ ).

\section{Efficiency of 3-presentations}

Recall that the deficiency of a finite presentation $<\underline{x} \mid \underline{r}>$ is the integer $|\underline{r}|-|\underline{x}|$. The deficiency of a finite group $G$, which we denote by $\operatorname{def}_{2}(G)$, is defined to be the least possible deficiency of any presentation of the group. P. Hall proved that every finite group $G$ satisfies

$$
\operatorname{def}_{2}(G) \geqslant d\left(H_{2}(G)\right) \text {, }
$$

where $d\left(H_{2}(G)\right)$ denotes the minimal cardinality of a generating set for the second integral homology group $H_{2}(G)$ (see for instance [3]). Using $\operatorname{def}_{1}(G)$ to denote the minimum cardinality of a generating set for $G$, the well-known isomorphism $H_{1}(G) \cong G^{a b}$ implies

$$
\operatorname{def}_{1}(G) \geqslant d\left(H_{1}(G)\right) .
$$

Recall that a presentation $<\underline{x} \mid \underline{r}>$ of a finite group $G$ is said to be efficient if $|\underline{r}|-|\underline{x}|=$ $d\left(H_{2}(G)\right)$. For instance [20],

$$
<x_{1}, \ldots, x_{n} \mid x_{1}^{m_{1}}, \ldots, x_{n}^{m_{n}}, x_{i} x_{j} x_{i}^{-1} x_{j}^{-1} \text { for } 1 \leqslant i \leqslant j \leqslant n>
$$

is an efficient presentation of an (arbitrary) finite abelian group if each $m_{i}$ divides $m_{i+1}$. Many finite groups admit no efficient presentation [31][24]. However, every finite group $G$ can be embedded in a finite group that does admit an efficient presentation [23].

We extend these ideas to 3-presentations as follows. 
Table 2: A summary of Appendix A

\begin{tabular}{|c|c|c|c|c|c|c|c|}
\hline Name & $|G|$ & Defining relators & $|\underline{s}|$ & $\mathrm{rk} \pi$ & $H_{1}(G)$ & $H_{2}(G)$ & $H_{3}(G)$ \\
\hline$D_{3}$ & 6 & $x^{2}, y^{3},(x y)^{2}$ & 3 & 11 & $\overline{\mathbf{Z}_{2}}$ & 0 & $\overline{\mathbf{Z}_{6}}$ \\
\hline$D_{4}$ & 8 & $x^{2}, y^{4},(x y)^{2}$ & 4 & 15 & $\left(\mathbf{Z}_{2}\right)^{2}$ & $\mathbf{Z}_{2}$ & $\left(\mathbf{Z}_{2}\right)^{2} \oplus \mathbf{Z}_{4}$ \\
\hline$Q_{2}$ & 8 & $x^{-2} y^{2}, x^{-2}(x y)^{2}$ & 1 & 7 & $\left(\mathbf{Z}_{2}\right)^{2}$ & 0 & $\mathbf{Z}_{8}$ \\
\hline$D_{5}$ & 10 & $x^{2}, y^{5},(x y)^{2}$ & 3 & 19 & $\mathbf{Z}_{2}$ & 0 & $\mathbf{z}_{10}$ \\
\hline$D_{6}$ & 12 & $x^{2}, y^{6},(x y)^{2}$ & 4 & 23 & $\left(\mathbf{Z}_{2}\right)^{2}$ & $\mathbf{Z}_{2}$ & $\left(\mathbf{Z}_{2}\right)^{3} \oplus \mathbf{Z}_{3}$ \\
\hline$A_{4}$ & 12 & $x^{3}, y^{2},(x y)^{3}$ & 3 & 23 & $\mathbf{Z}_{3}$ & $\mathbf{z}_{2}$ & $\mathbf{z}_{6}$ \\
\hline$Q_{3}$ & 12 & $x^{-2} y^{3}, x^{-2}(x y)^{2}$ & 1 & 11 & $\mathbf{z}_{2}$ & 0 & $\mathbf{z}_{12}$ \\
\hline$D_{7}$ & 14 & $x^{2}, y^{7},(x y)^{2}$ & 3 & 27 & $\mathbf{z}_{2}$ & 0 & $\mathbf{z}_{14}$ \\
\hline$C_{2} \times D_{4}$ & 16 & $\begin{array}{l}x^{2}, y^{2}, z^{2} \\
(y z)^{4},(x y)^{2},(x z)^{2}\end{array}$ & 10 & 63 & $\left(\mathbf{Z}_{2}\right)^{3}$ & $\left(\mathbf{Z}_{2}\right)^{3}$ & $\left(\mathbf{Z}_{2}\right)^{6} \oplus \mathbf{Z}_{4}$ \\
\hline$C_{2} \times Q_{2}$ & 16 & $\begin{array}{l}x^{-2} y^{2}, x^{-2}(x y)^{2} \\
z^{2},(x z)^{2},(y z)^{2}\end{array}$ & 6 & 47 & $\left(\mathbf{Z}_{2}\right)^{3}$ & $\left(\mathbf{Z}_{2}\right)^{2}$ & $\left(\mathbf{Z}_{2}\right)^{3} \oplus \mathbf{Z}_{8}$ \\
\hline \multirow[t]{6}{*}{$D_{8}$} & 16 & $x^{2}, y^{8},(x y)^{2}$ & 4 & 31 & $\left(\mathbf{Z}_{2}\right)^{2}$ & $\mathbf{Z}_{2}$ & $\left(\mathbf{Z}_{2}\right)^{2} \oplus \mathbf{Z}_{8}$ \\
\hline & 16 & $x^{2}, x y x y^{-3}$ & 2 & 15 & $\left(\mathbf{Z}_{2}\right)^{2}$ & 0 & $\mathbf{Z}_{2} \oplus \mathbf{Z}_{8}$ \\
\hline & 16 & $x^{2}, x y x y^{3}$ & 2 & 15 & $\mathbf{Z}_{2} \oplus \mathbf{Z}_{4}$ & 0 & $\mathbf{Z}_{2} \oplus \mathbf{Z}_{8}$ \\
\hline & 16 & $x^{4}, y^{4}, x^{-1} y x y$ & 4 & 31 & $\mathbf{Z}_{2} \oplus \mathbf{Z}_{4}$ & $\mathbf{Z}_{2}$ & $\left(\mathbf{Z}_{4}\right)^{2} \oplus \mathbf{Z}_{2}$ \\
\hline & 16 & $x^{4}, y^{4},(x y)^{2},\left(x^{-1} y\right)^{2}$ & 6 & 47 & $\mathbf{Z}_{2} \oplus \mathbf{Z}_{4}$ & $\left(\mathbf{Z}_{2}\right)^{2}$ & $\left(\mathbf{Z}_{4}\right)^{2} \oplus\left(\mathbf{Z}_{2}\right)^{2}$ \\
\hline & 16 & $\begin{array}{l}x^{2}, y^{2}, z^{2}, x y z(y z x)^{-1} \\
x y z(z x y)^{-1}\end{array}$ & 6 & 47 & $\left(\mathbf{Z}_{2}\right)^{3}$ & $\left(\mathbf{Z}_{2}\right)^{2}$ & $\left(\mathbf{Z}_{2}\right)^{3} \oplus \mathbf{Z}_{8}$ \\
\hline$Q_{4}$ & 16 & $x^{-2} y^{4}, x^{-2}(x y)^{2}$ & 1 & 15 & $\left(\mathbf{Z}_{2}\right)^{2}$ & 0 & $\mathbf{z}_{16}$ \\
\hline$C_{3} \times D_{3}$ & 18 & $x^{2}, y^{3},(x y)^{2}(y x)^{-2}$ & 3 & 35 & $\mathbf{z}_{6}$ & 0 & $\mathbf{Z}_{3} \oplus \mathbf{Z}_{6}$ \\
\hline \multirow[t]{2}{*}{$D_{9}$} & 18 & $x^{2}, y^{9},(x y)^{2}$ & 3 & 35 & $\mathbf{z}_{2}$ & 0 & $\mathbf{Z}_{18}$ \\
\hline & 18 & $\begin{array}{l}x^{2}, y^{2}, z^{2},(x y)^{3}, \\
(x z)^{3},(x y z)^{2}\end{array}$ & 7 & 71 & $\mathbf{Z}_{2}$ & $\mathbf{z}_{3}$ & $\left(\mathbf{Z}_{3}\right)^{3} \oplus \mathbf{Z}_{2}$ \\
\hline \multirow[t]{2}{*}{$D_{10}$} & 20 & $x^{2}, y^{10},(x y)^{2}$ & 4 & 39 & $\left(\mathbf{Z}_{2}\right)^{2}$ & $\mathbf{Z}_{2}$ & $\left(\mathbf{Z}_{2}\right)^{3} \oplus \mathbf{Z}_{5}$ \\
\hline & 20 & $x^{5}, y^{4}, x^{2} y^{-1} x^{-1} y$ & 3 & 39 & $\mathbf{Z}_{4}$ & 0 & $\mathbf{Z}_{4}$ \\
\hline \multirow[t]{2}{*}{$Q_{5}$} & 20 & $x^{-2} y^{5}, x^{-2}(x y)^{2}$ & 1 & 19 & $\mathbf{Z}_{2}$ & 0 & $\mathbf{Z}_{20}$ \\
\hline & 21 & $x^{3}, y^{2} x^{-1} y^{-1} x$ & 2 & 20 & $\mathbf{z}_{3}$ & 0 & $\mathbf{Z}_{3}$ \\
\hline$D_{11}$ & 22 & $x^{2}, y^{11},(x y)^{2}$ & 3 & 43 & $\mathbf{z}_{2}$ & 0 & $\mathbf{z}_{22}$ \\
\hline$C_{2} \times A_{4}$ & 24 & $x^{3}, y^{2},\left(x^{-1} y x y\right)^{2}$ & 5 & 47 & $\mathbf{z}_{6}$ & $\mathbf{Z}_{2}$ & $\left(\mathbf{Z}_{2}\right)^{3} \oplus \mathbf{Z}_{3}$ \\
\hline$C_{2} \times D_{6}$ & 24 & $\begin{array}{l}x^{2}, y^{2}, z^{2},(y z)^{6} \\
(x y)^{2},(x z)^{2}\end{array}$ & 10 & 95 & $\left(\mathbf{Z}_{2}\right)^{3}$ & $\left(\mathbf{z}_{2}\right)^{3}$ & $\left(\mathbf{Z}_{2}\right)^{7} \oplus \mathbf{Z}_{3}$ \\
\hline$C_{3} \times D_{4}$ & 24 & $x^{12}, y^{2}, y x y x^{5}$ & 4 & 47 & $\mathbf{Z}_{2} \oplus \mathbf{Z}_{6}$ & $\mathbf{Z}_{2}$ & $\left(\mathbf{Z}_{2}\right)^{2} \oplus \mathbf{Z}_{12}$ \\
\hline$C_{3} \times Q_{2}$ & 24 & $x^{12}, y^{2} x^{-6}, y^{-1} x y x^{-7}$ & 3 & 47 & $\mathbf{Z}_{2} \oplus \mathbf{Z}_{6}$ & 0 & $\mathbf{Z}_{24}$ \\
\hline$C_{4} \times D_{3}$ & 24 & $x^{12}, y^{2}, y x y x^{-5}$ & 4 & 47 & $\mathbf{Z}_{2} \oplus \mathbf{Z}_{4}$ & $\mathbf{Z}_{2}$ & $\left(\mathbf{Z}_{2}\right)^{2} \oplus \mathbf{Z}_{12}$ \\
\hline$C_{2} \times Q_{3}$ & 24 & $x^{6}, y^{4}, y^{-1} x y x$ & 4 & 47 & $\mathbf{Z}_{2} \oplus \mathbf{Z}_{4}$ & $\mathbf{Z}_{2}$ & $\left(\mathbf{Z}_{2}\right)^{2} \oplus \mathbf{Z}_{12}$ \\
\hline$D_{12}$ & 24 & $x^{2}, y^{12},(x y)^{2}$ & 4 & 47 & $\left(\mathbf{Z}_{2}\right)^{2}$ & $\mathbf{z}_{2}$ & $\left(\mathbf{Z}_{2}\right)^{2} \oplus \mathbf{Z}_{12}$ \\
\hline$S_{4}$ & 24 & $x^{4}, y^{2},(x y)^{3}$ & 4 & 47 & $\mathbf{z}_{2}$ & $\mathbf{z}_{2}$ & $\mathbf{Z}_{2} \oplus \mathbf{Z}_{12}$ \\
\hline \multirow[t]{3}{*}{$\hat{A}_{4}$} & 24 & $x^{-3} y^{3}, x^{-2} y x y$ & 1 & 23 & $\mathbf{z}_{3}$ & 0 & $\mathbf{Z}_{24}$ \\
\hline & 24 & $x^{4}, y^{6},(x y)^{2},\left(x^{-1} y\right)^{2}$ & 5 & 71 & $\left(\mathbf{Z}_{2}\right)^{2}$ & $\mathbf{Z}_{2}$ & $\left(\mathbf{Z}_{2}\right)^{2} \oplus \mathbf{Z}_{12}$ \\
\hline & 24 & $x^{-2} y^{2}, x^{-2}(x y)^{3}$ & 1 & 23 & $\mathbf{Z}_{8}$ & 0 & $\mathbf{Z}_{24}$ \\
\hline$Q_{6}$ & 24 & $x^{-2} y^{6}, x^{-2}(x y)^{2}$ & 1 & 23 & $\left(\mathbf{Z}_{2}\right)^{2}$ & 0 & $\mathbf{Z}_{24}$ \\
\hline$D_{13}$ & 26 & $x^{2}, y^{13},(x y)^{2}$ & 3 & 51 & $\mathbf{Z}_{2}$ & 0 & $\mathbf{Z}_{26}$ \\
\hline \multirow[t]{2}{*}{$B(2,3)$} & 27 & $x^{3}, y^{3},(x y)^{3},\left(x^{-1} y\right)^{3}$ & 6 & 80 & $\left(\mathbf{Z}_{3}\right)^{2}$ & $\left(\mathbf{Z}_{3}\right)^{2}$ & $\left(\mathbf{Z}_{3}\right)^{4}$ \\
\hline & 27 & $x^{3}, x^{-1} y x y^{2}$ & 2 & 26 & $\left(\mathbf{Z}_{3}\right)^{2}$ & 0 & $\left(\mathbf{Z}_{3}\right)^{2}$ \\
\hline$D_{14}$ & 28 & $x^{2}, y^{14},(x y)^{2}$ & 4 & 55 & $\left(\mathbf{Z}_{2}\right)^{2}$ & $\mathbf{Z}_{2}$ & $\left(\mathbf{Z}_{2}\right)^{2} \oplus \mathbf{Z}_{14}$ \\
\hline$Q_{7}$ & 28 & $x^{-2} y^{7}, x^{-2}(x y)^{2}$ & 1 & 27 & $\mathbf{Z}_{4}$ & 0 & $\mathbf{Z}_{28}$ \\
\hline$C_{3} \times D_{5}$ & 30 & $x^{2}, x y x y^{-4}$ & 2 & 29 & $\mathbf{z}_{6}$ & 0 & $\mathbf{Z}_{30}$ \\
\hline$C_{5} \times D_{3}$ & 30 & $x^{2}, x y x y^{4}$ & 2 & 29 & $\mathbf{Z}_{10}$ & 0 & $\mathbf{Z}_{30}$ \\
\hline$D_{15}$ & 30 & $x^{2}, y^{15},(x y)^{2}$ & 3 & 59 & $\mathbf{z}_{2}$ & 0 & $\mathbf{z}_{30}$ \\
\hline
\end{tabular}


Definition 3. Let the deficiency of a 3-presentation $<\underline{x}|\underline{r}| \underline{s}>$ be the integer $|\underline{s}|-|\underline{r}|+|\underline{x}|$. The 3-deficiency of a finite group $G$ is then taken to be the least possible deficiency of any 3 -presentation of the group, and will be denoted by $\operatorname{def}_{3}(G)$.

Proposition 4. The inequality

$$
\operatorname{def}_{3}(G) \geqslant d\left(H_{3}(G)\right)
$$

holds for every finite group $G$.

Proof. Let $<\underline{x}|\underline{r}| \underline{s}>$ be some 3-presentation of the finite group $G$, and let $X$ be the associated 3 -dimensional $\mathrm{CW}$-space. The integral homology groups $H_{n}(X)$ are the homology groups of a chain complex of free abelian groups

$$
0 \rightarrow \oplus_{\underline{s}} \mathbf{Z} \rightarrow \oplus_{\underline{r}} \mathbf{Z} \rightarrow \oplus_{\underline{x}} \mathbf{Z} \rightarrow 0 .
$$

By a well-known result on the Euler-Poincaré characteristic [29, page 146] we have

$$
|\underline{s}|-|\underline{r}|+|\underline{x}|=\operatorname{rank}_{\mathbf{Z}} H_{3}(X)-\operatorname{rank}_{\mathbf{Z}} H_{2}(X)+\operatorname{rank}_{\mathbf{Z}} H_{1}(X) .
$$

But $H_{2}(X)=H_{2}(G), H_{1}(X)=H_{1}(G)$ are finite groups and thus have zero rank. Therefore

$$
|\underline{s}|-|\underline{r}|+|\underline{x}|=\operatorname{rank}_{\mathbf{Z}} H_{3}(X) .
$$

The proposition follows from the obvious inequality $\operatorname{rank}_{\mathbf{Z}} H_{3}(X) \geqslant d\left(H_{3}(G)\right)$.

Definition 4. We say that a 3-presentation $<\underline{x}|\underline{r}| \underline{s}>$ of a finite group $G$ is efficient when $|\underline{s}|-|\underline{r}|+|\underline{x}|=d\left(H_{3}(G)\right)$.

The efficient 2-presentation of a finite abelian group given above can be extended, using Proposition 2, to a 3-presentation. One can check that the resulting 3-presentation is efficient. This proves the following proposition.

Proposition 5. Every finite abelian group admits an efficient 3-presentation.

We now adapt ideas from [31] and [23] to show that not all finite groups admit efficient 3 -presentations, but that all finite $p$-groups embed into a finite $p$-group that does admit an efficient 3-presentation. (It seems to be a difficult problem to decide whether, in fact, every finite $p$-group admits an efficient 3-presentation.) In a future paper we shall prove that an arbitrary finite group embeds into a finite group admitting an efficient 3-presentation.

Proposition 6. Let $G$ be a d-generator group of prime power order $p^{n}$. Suppose that the Frattini subgroup $\Phi(G)$ is a $t$-generator group. Then

$$
\begin{gathered}
\operatorname{def}_{2}(G) \geqslant \frac{d(d-1)}{2}-t, \\
\operatorname{def}_{3}(G) \geqslant \frac{d\left(d^{2}+5\right)}{6}-n t,
\end{gathered}
$$

and any 3-presentation $<\underline{x}|\underline{r}| \underline{s}>$ satisfies

$$
\begin{gathered}
|\underline{x}| \geqslant d, \\
|\underline{r}| \geqslant \frac{d(d+1)}{2}-t,
\end{gathered}
$$




$$
|\underline{s}| \geqslant \frac{d(d+1)(d+2)}{6}-t(n+1) .
$$

Proof. There is an exact homology sequence [14]

$$
\begin{aligned}
& H_{3}(G) \rightarrow H_{3}(G / \Phi(G)) \rightarrow \operatorname{ker}(\Phi(G) \wedge G \rightarrow G) \\
& \rightarrow H_{2}(G) \rightarrow H_{2}(G / \Phi(G)) \rightarrow \Phi(G) /[\Phi(G), G]
\end{aligned}
$$

where $\wedge$ denotes a certain nonabelian exterior product. A bound

$$
|\Phi(G) \wedge G| \leqslant p^{t n}
$$

is given in [18], and we have [17]:

$$
\begin{aligned}
& d\left(H_{2}(G / \Phi(G))\right)=\frac{d(d-1)}{2}, \\
& d\left(H_{3}(G / \Phi(G))\right)=\frac{d\left(d^{2}+5\right)}{6} .
\end{aligned}
$$

The inequalities of the proposition follow from this exact sequence, bound, equalities, Proposition 4 and P. Hall's inequality for $\operatorname{def}_{2}(G)$.

This result is of interest for $p$-groups whose Frattini subgroup has a small generating set. For example, it implies that any 3-presentation $\langle\underline{x}|\underline{r}| \underline{s}\rangle$ of an extra-special group $G$ of order $p^{n}$ (thus [3] $\left.n=2 k+1, t=1, d=2 k\right)$ needs $|\underline{s}| \geqslant\left(d^{3}+3 d^{2}-4 d-6\right) / 6$.

In [31] a certain finite group $G$ was shown to admit no efficient 2-presentation by considering a subgroup $H$ of index $|G: H|=k$ and using the formula $\left(\operatorname{def}_{2}(G)+1\right) k \geqslant$ $\operatorname{def}_{2}(H)+1$. This formula follows from the Reidemeister-Schreier Theorem. The formula extends to 3-presentations as follows.

Proposition 7. Let $G$ be a finite group with subgroup $H$ of index $|G: H|=k$. Then

$$
\operatorname{def}_{3}(G)-1 \geqslant \frac{1}{k}\left(\operatorname{def}_{3}(H)-1\right) .
$$

Proof. Let $<\underline{x}|\underline{r}| \underline{s}>$ be a 3-presentation of $G$ with $|\underline{s}|-|\underline{r}|+|\underline{x}|=\operatorname{def}_{3}(G)$. Let $F=F(\underline{x})$ be the free group on $\underline{x}$, let $R$ be the normal subgroup of $F$ generated by the relators $\underline{r}$, and let $F_{H}$ be the preimage in $F$ of the subgroup $H \leqslant F / R$. Thus $\left|F: F_{H}\right|=k$. The free crossed $F$-module $\partial: C(\underline{r}) \rightarrow F$ restricts to a crossed $F_{H}$-module $\partial_{H}: C(\underline{r}) \rightarrow F_{H}$. As explained in [19], $\partial_{H}$ is the free crossed $F_{H}$-module on the function $T \times \underline{r} \rightarrow F_{H},(t, r) \mapsto t r t^{-1}$ where $T$ is a transversal of $F_{H}$ in $F$. Hence $\partial_{H}$ is the crossed module arising from some presentation $\left\langle\underline{x}^{\prime} \mid \underline{r}^{\prime}\right\rangle$ of $H$. By the ReidemeisterSchreier theorem we have $\left|\underline{x}^{\prime}\right|=1+k(|\underline{x}|-1)$. Furthermore, $\left|\underline{r}^{\prime}\right|=|T| \times|\underline{r}|=k|\underline{r}|$. Now ker $\partial=\operatorname{ker} \partial_{H}$ is generated as a $\mathbf{Z} H$-module by $|T| \times|\underline{s}|=k|\underline{s}|$ elements, and so the presentation $<\underline{x}^{\prime} \mid \underline{r}^{\prime}>$ can be extended to a 3-presentation $<\underline{x}^{\prime}\left|\underline{r}^{\prime}\right| \underline{s}^{\prime}>$ of $H$ with $\left|\underline{s^{\prime}}\right|=k|\underline{s}|$. Consequently

$$
\begin{aligned}
k\left(\operatorname{def}_{3}(G)-1\right) & =k(|\underline{s}|-|\underline{r}|+|\underline{x}|-1) \\
& =\left|\underline{s}^{\prime}\right|-\left|\underline{r}^{\prime}\right|+\left|\underline{x}^{\prime}\right|-1 \\
& \geqslant \operatorname{def}_{3}(H)-1 .
\end{aligned}
$$


Proposition 8. The group $A_{4}$ admits an efficient 2-presentation, but admits no efficient 3-presentation.

Proof. An efficient 2-presentation for $A_{4}$ is exhibited in Table 2. The group $A_{4}$ has a

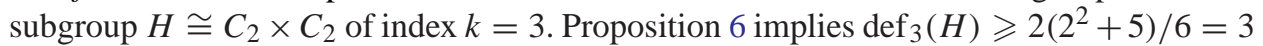
(in fact, equality holds here). So Proposition 7 implies that $\operatorname{def}_{3}\left(A_{4}\right) \geqslant\left(\operatorname{def}_{3}(H)-1\right) / 3+$ $1=5 / 3$. Table 2 shows that $d\left(H_{3}\left(A_{4}\right)\right)=1$. Hence $\operatorname{def}_{3}\left(A_{4}\right) \neq d\left(H_{3}\left(A_{4}\right)\right)$.

The preceding proof shows that the 3 -presentation for $A_{4}$ given in Table 2 is minimal in the sense that no other 3-presentation has smaller deficiency. It was mentioned in Section 3 that $S_{3}$ admits an efficient 2-presentation. We suspect (but have not proved) that $S_{3}$ admits no efficient 3-presentation.

A result of R.G. Swan ([31, Corollary 5.2]) immediately implies the following useful result on prime-power groups.

Proposition 9. [31] Let $G$ be a d-generator finite p-group that admits an efficient 2presentation involving just d generators. Then $G$ admits an efficient 3-presentation.

It is an unsolved problem as to whether every finite $p$-group satisfies the hypothesis of Proposition 9 ( $c f$. [26]). J. Harlander [23] has shown that for any finite group $G$ and any prime $p$ there exists an integer $n \geqslant 0$ such that the group $G \times \prod_{i=1}^{n} C_{p}$ is a $d$-generator $p$ group which admits an efficient 2-presentation on $d$ generators. In particular, this means that any finite $p$-group embeds into a finite $p$-group that satisfies the hypothesis of Proposition 9. The proposition thus yields the following further proposition.

Proposition 10. Any finite p-group embeds into a finite p-group that admits an efficient 3-presentation.

\section{Cohomology}

Let $\langle\underline{x}| \underline{r}>$ be a presentation of a group $G$, and let $\pi=\operatorname{ker} \Delta$ be the $\mathbf{Z} G$-module of identities for this presentation. The cohomology $H^{n}(G, A)$ of $G$ with coefficients in a $\mathbf{Z} G$-module $A$ has a well-known description in terms of free $\mathbf{Z} G$-resolutions of $\mathbf{Z}$. It is a routine exercise to extract from this description the following isomorphisms

$$
\begin{gathered}
H^{2}(G, A) \cong \frac{\operatorname{ker}\left(\operatorname{Hom}_{\mathbf{Z} G}\left(\oplus_{\underline{r}} \mathbf{Z} G, A\right) \rightarrow \operatorname{Hom}_{\mathbf{Z} G}(\pi, A)\right)}{\operatorname{im}\left(\operatorname{Hom}_{\mathbf{Z} G}\left(\oplus_{\underline{x}} \mathbf{Z} G, A\right) \rightarrow \operatorname{Hom}_{\mathbf{Z} G}\left(\oplus_{\underline{r}} \mathbf{Z} G, A\right)\right)}, \\
H^{3}(G, A) \cong \operatorname{coker}\left(\operatorname{Hom}_{\mathbf{Z} G}\left(\oplus_{\underline{r}} \mathbf{Z} G, A\right) \rightarrow \operatorname{Hom}_{\mathbf{Z} G}(\pi, A)\right) .
\end{gathered}
$$

These isomorphisms enable one to compute the cohomology groups in dimensions $n=2,3$ from a knowledge of the module $\pi$. Of course, even for $n \geqslant 4$ the cohomology $H^{n}(G, A)$ can be expressed in terms of $\pi$ by means of the following formula:

$$
H^{n}(G, A) \cong \operatorname{Ext}_{\mathbf{Z} G}^{n-3}(\pi, A), \quad n \geqslant 4 .
$$

As explained in the Introduction (Section 1), it is the third cohomology group $H^{3}(G, A)$ that is of particular interest to us. So for the remainder of this section we consider in more detail the problem of computing this cohomology group under the simplifying assumption that $G$ acts trivially on $A$. 
When $G$ acts trivially on $A$, the Universal Coefficient Theorem [29, page 385] states that

$$
H^{3}(G, A) \cong \operatorname{Ext}\left(H_{2}(G), A\right) \oplus \operatorname{Hom}_{\mathbf{Z}}\left(H_{3}(G), A\right),
$$

where $H_{n}(G)$ denotes the integral homology group $H_{n}(G, \mathbf{Z})$. Since the functors Ext $(-,-)$ and $\operatorname{Hom}_{\mathbf{Z}}(-,-)$ are routine to evaluate, the computation of $H^{3}(G, A)$ reduces to the problem of computing $\mathrm{H}_{2}(G)$ and $\mathrm{H}_{3}(G)$.

From a 3-presentation $<\underline{x}|\underline{r}| \underline{s}>$ of $G$ we can construct the following commutative diagram of $\mathbf{Z} G$-module homomorphisms, in which the rows are short exact and the middle column is exact.

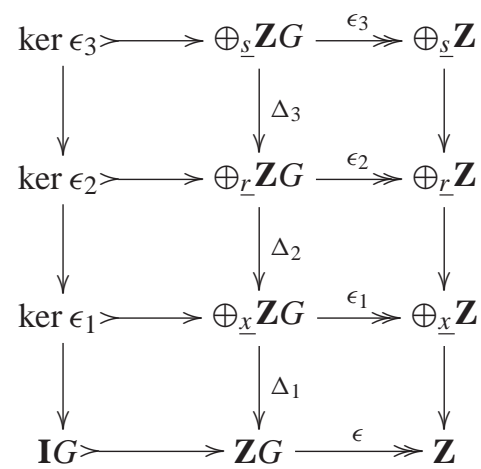

Here $\epsilon$ is the augmentation map, and each $\epsilon_{i}$ is the canonical map induced by $\epsilon$. The map $\Delta_{2}$ is the map $\Delta$ (described in Section 2). The map $\Delta_{3}$ sends the basis elements (which are in one-one correspondence with $\underline{s}$ ) to the corresponding generators of ker $\Delta_{2}$. The map $\Delta_{1}$ sends the basis element corresponding to $x_{i} \in \underline{x}$ to the element $x_{i}-1 \in \mathbf{Z} G$.

We view the above diagram as the bottom part of a short exact sequence of chain complexes

$$
B_{*} \longrightarrow C_{*} \longrightarrow D_{*}
$$

in which $B_{n}=C_{n}=D_{n}=0$ for $n \leqslant-1$, and $H_{n}\left(C_{*}\right)=0, H_{n}\left(D_{*}\right) \cong H_{n}(G)$ for $n \geqslant 1$. The resulting exact homology sequence

$$
\cdots \rightarrow H_{3}\left(C_{*}\right) \rightarrow H_{3}\left(D_{*}\right) \rightarrow H_{2}\left(B_{*}\right) \rightarrow H_{2}\left(C_{*}\right) \rightarrow \cdots
$$

implies that

$$
\begin{aligned}
& H_{2}(G) \cong H_{1}\left(B_{*}\right) \cong \frac{\operatorname{ker}\left(\epsilon_{1}\right) \cap \operatorname{ker}\left(\Delta_{1}\right)}{\Delta_{2}\left(\operatorname{ker} \epsilon_{2}\right)}, \\
& H_{3}(G) \cong H_{2}\left(B_{*}\right) \cong \frac{\operatorname{ker}\left(\epsilon_{2}\right) \cap \operatorname{ker}\left(\Delta_{2}\right)}{\Delta_{3}\left(\operatorname{ker} \epsilon_{3}\right)} .
\end{aligned}
$$

When $G$ is finite, GAP's LLL algorithm [21] [27] can be used to find a $\mathbf{Z}$-basis for the modules $\operatorname{ker}\left(\epsilon_{1}\right) \cap \operatorname{ker}\left(\Delta_{1}\right)$ and $\operatorname{ker}\left(\epsilon_{2}\right) \cap \operatorname{ker}\left(\Delta_{2}\right)$.

In order to find $\mathbf{Z}$-bases for the modules $\Delta_{2}\left(\operatorname{ker} \epsilon_{2}\right)$ and $\Delta_{3}\left(\operatorname{ker} \epsilon_{3}\right)$ we abuse the notation slightly and let $\underline{r}, \underline{s}$ denote generating sets for the $\mathbf{Z} G$-modules ker $\Delta_{1}$, ker $\Delta_{2}$. The sets $S_{2}=\{(g-1) a: g \in G, a \in \underline{r}\} S_{3}=\{(g-1) w: g \in G, w \in \underline{s}\}$ then generate ker $\epsilon_{1}$ and ker $\epsilon_{2}$ as abelian groups. Thus the LLL algorithm can be applied to $S_{2}$ and $S_{3}$ to find $\mathbf{Z}$-bases for $\Delta_{2}\left(\operatorname{ker} \epsilon_{2}\right)$ and $\Delta_{3}\left(\operatorname{ker} \epsilon_{3}\right)$.

Given the appropriate $\mathbf{Z}$-bases, it is routine to determine the abelian groups $\mathrm{H}_{2}(G)$ and $H_{3}(G)$ from isomorphisms (III) and (IV). (We remark that three alternative methods for 
computing $H_{2}(G)$ are described and implemented in [16].)

We illustrate the above discussion by computing $H_{3}(G)$ for $G=S_{3}$. Let us resume the notation of Section 3. Then $\operatorname{ker}\left(\epsilon_{2}\right) \cap \operatorname{ker}\left(\Delta_{2}\right)$ is the nullspace of the following matrix:

$$
E=\left(\begin{array}{cccccccccccccccccc}
1 & 1 & 1 & 1 & 1 & 1 & 0 & 0 & 0 & 0 & 0 & 0 & 0 & 0 & 0 & 0 & 0 & 0 \\
0 & 0 & 0 & 0 & 0 & 0 & 1 & 1 & 1 & 1 & 1 & 1 & 0 & 0 & 0 & 0 & 0 & 0 \\
0 & 0 & 0 & 0 & 0 & 0 & 0 & 0 & 0 & 0 & 0 & 0 & 1 & 1 & 1 & 1 & 1 & 1 \\
1 & 1 & 0 & 0 & 0 & 0 & 0 & 0 & 0 & 0 & 0 & 0 & 1 & 0 & 0 & 0 & 1 & 0 \\
1 & 1 & 0 & 0 & 0 & 0 & 0 & 0 & 0 & 0 & 0 & 0 & 0 & 1 & 1 & 0 & 0 & 0 \\
0 & 0 & 1 & 0 & 0 & 1 & 0 & 0 & 0 & 0 & 0 & 0 & 0 & 1 & 1 & 0 & 0 & 0 \\
0 & 0 & 0 & 1 & 1 & 0 & 0 & 0 & 0 & 0 & 0 & 0 & 0 & 0 & 0 & 1 & 0 & 1 \\
0 & 0 & 0 & 1 & 1 & 0 & 0 & 0 & 0 & 0 & 0 & 0 & 1 & 0 & 0 & 0 & 1 & 0 \\
0 & 0 & 1 & 0 & 0 & 1 & 0 & 0 & 0 & 0 & 0 & 0 & 0 & 0 & 0 & 1 & 0 & 1 \\
0 & 0 & 0 & 0 & 0 & 0 & 1 & 0 & 1 & 1 & 0 & 0 & 0 & 1 & 1 & 0 & 0 & 0 \\
0 & 0 & 0 & 0 & 0 & 0 & 0 & 1 & 0 & 0 & 1 & 1 & 1 & 0 & 0 & 0 & 1 & 0 \\
0 & 0 & 0 & 0 & 0 & 0 & 1 & 0 & 1 & 1 & 0 & 0 & 0 & 0 & 0 & 1 & 0 & 1 \\
0 & 0 & 0 & 0 & 0 & 0 & 1 & 0 & 1 & 1 & 0 & 0 & 1 & 0 & 0 & 0 & 1 & 0 \\
0 & 0 & 0 & 0 & 0 & 0 & 0 & 1 & 0 & 0 & 1 & 1 & 0 & 0 & 0 & 1 & 0 & 1 \\
0 & 0 & 0 & 0 & 0 & 0 & 0 & 1 & 0 & 0 & 1 & 1 & 0 & 1 & 1 & 0 & 0 & 0
\end{array}\right) .
$$

If the matrix $E$ is entered into the GAP package, the command

$$
\mathrm{U}:=\text { LLLReducedBasis(TransposedMat(E), "linearcomb").relations; }
$$

produces the following $\mathbf{Z}$-basis for $\operatorname{ker}\left(\epsilon_{2}\right) \cap \operatorname{ker}\left(\Delta_{2}\right)$ :

$$
\begin{array}{llll}
u_{1}= & (-1,1,0,0,0,0, & 0,0,0,0,0,0, & 0,0,0,0,0,0), \\
u_{2}=(0,0,0,-1,1,0, & 0,0,0,0,0,0, & 0,0,0,0,0,0), \\
u_{3}=(0,0,-1,0,0,1, & 0,0,0,0,0,0, & 0,0,0,0,0,0), \\
u_{4}=(0,0,0,0,0,0, & -1,0,1,0,0,0, & 0,0,0,0,0,0), \\
u_{5}=(0,0,0,0,0,0, & -1,0,0,1,0,0, & 0,0,0,0,0,0), \\
u_{6}= & (0,0,0,0,0,0, & 0,-1,0,0,1,0, & 0,0,0,0,0,0), \\
u_{7}= & (0,0,0,0,0,0, & 0,-1,0,0,0,1, & 0,0,0,0,0,0), \\
u_{8}= & (0,0,0,0,0,0, & 0,0,0,0,0,0, & 0,-1,1,0,0,0), \\
u_{9}=(0,0,0,0,0,0, & 0,0,0,0,0,0, & -1,0,0,0,1,0), \\
u_{10}=(0,0,0,0,0,0, & 0,0,0,0,0,0, & 0,0,0,-1,0,1) .
\end{array}
$$

If the 20 non-zero vectors $g . v_{i}-v_{i}$ (where $g \in G, i \in\{1,2,5,9\}$ and the $v_{i}$ are the vectors of Section 3) are entered into GAP as the rows of a vector $W$, the command

$$
\mathrm{W}:=\text { LLLReducedBasis(W).basis; }
$$

converts $W$ into a matrix having 10 linearly independent rows, each row being an integer linear combination of the above vectors $u_{i}$. For each $1 \leqslant j \leqslant 10$ the two commands

$\mathrm{U}[11]:=\mathrm{W}[j] ; \quad \mathrm{W}:=$ LLLReducedBasis(U, "linearcomb").relations; express the $j$ th row $w_{j}$ of $W$ as a linear combination of the $u_{i}$. We find that the quotient

$$
H=<u_{1}, \ldots, u_{10}>/<w_{1}, \ldots, w_{10}>
$$


is an abelian group with generators $u_{1}, \ldots, u_{10}$ subject to the relations:

$$
\begin{gathered}
u_{8}-u_{10}=0, u_{9}+u_{10}=0,-u_{9}+u_{10}=0,-u_{2}-u_{10}=0,-u_{1}+u_{10}=0, \\
-u_{3}-u_{10}=0, u_{5}+u_{6}=0,-u_{4}+u_{5}+u_{6}-u_{7}=0,-u_{4}+u_{6}=0, u_{4}-u_{5}+u_{6}=0 .
\end{gathered}
$$

The group $H$ can be entered into GAP as a finitely presented (nonabelian) group. The command

$$
\mathrm{H}:=\text { Abelianquotients(H); }
$$

then recovers the well-known result that $H_{3}\left(S_{3}\right) \cong H=\mathbf{Z}_{6}$.

\section{Twisted coefficients}

If the group $G$ acts non-trivially on the module $A$, then the Universal Coefficient Theorem cannot be invoked, and the computations become slightly more unwieldy. Nevertheless, in principle a 3-presentation of $G$ can still be used to compute $H^{n}(G, A)$ for $0 \leqslant n \leqslant 3$, for any finite $G$, and any finitely generated $\mathbf{Z} G$-module $A$. We explain the method for $n=3$. As there are a number of ways of representing the module $A$ on a computer, the method for computing cohomology needs to vary accordingly.

We suppose that $A$ is presented by a short exact sequence of $\mathbf{Z} G$-modules

$$
0 \rightarrow B \rightarrow M \rightarrow A \rightarrow 0
$$

in which $M$ is a free $\mathbf{Z} G$-module. This data is captured by a set $\underline{m}$ of elements that freely generate the module $M$, together with any set $\underline{b}$ of elements that generate the ideal $B$. Each element in $\underline{b}$ is thus a $\mathbf{Z} G$-linear combination of elements of $\underline{m}$. We make use of the fact that $B$ has an underlying free abelian group.

Since $M$ is $\mathbf{Z} G$-free, the cohomology coefficient sequence implies an isomorphism $H^{3}(G, A) \cong H^{2}(G, B)$. For any set $y$ there is a canonical isomorphism of modules $\operatorname{Hom}_{\mathbf{Z} G}\left(\oplus_{\underline{y}} \mathbf{Z} G, A\right) \cong \oplus_{\underline{y}} A$. Thus, from equation (I) above, we obtain the isomorphism

$$
H^{3}(G, A) \cong \frac{\operatorname{ker} \alpha}{\operatorname{im} \beta}
$$

where $\alpha$ and $\beta$ are the homomorphisms

$$
\begin{aligned}
& \alpha: \bigoplus_{\underline{r}} B \longrightarrow \oplus_{\underline{s}} B, \\
& \beta: \oplus_{\underline{x}} B \longrightarrow \oplus_{\underline{r}} B,
\end{aligned}
$$

canonically induced by the 3-presentation $<\underline{x}|\underline{r}| \underline{s}>$ for $G$.

The LLL algorithm can be applied to the data $<\underline{m} \mid \underline{b}>$ to find a basis for the free abelian group underlying $B$. This enables one to view $\alpha$ and $\beta$ as homomorphisms of free abelian groups and, by copying the procedure in Section 9, to determine the finite abelian group $H^{3}(G, A) \cong \operatorname{ker} \alpha / \operatorname{im} \beta$.

The most crucial parameter for assessing the practicality of this computation of $H^{3}(G, A)$ is the $\mathbf{Z}$-rank of $\oplus_{\underline{r}} B$. Now $\operatorname{dim}_{\mathbf{Z}}(B) \leqslant \operatorname{dim}_{\mathbf{Z}}(M)=|\underline{m}| \times|G|$. The CPU times listed in Section 4 suggest that LLL algorithm is able to handle square matrices with 2000 rows. Thus, the computation should be practical for, say,

$$
|\underline{r}| \times|\underline{m}| \times|G| \leqslant 2000 \text {. }
$$


In future work we hope to increase this heuristic upper bound by incorporating the group structure of $G$ into more stages of the computation.

\section{Computer functions}

The data in Section 7 was compiled with the aid of six computer functions written in the computer algebra language GAP v.3 [21]. These functions automate the techniques explained above, and are contained in the text-file in Appendix B. The functions are made operational by saving the text-file under the name homology . gap and using the command

$$
\text { Read("homology.gap"); }
$$

from within the GAP v.3 system. Brief descriptions of the functions follow.

\section{FoxMatrix(G)}

Given a finitely presented finite group $G=<\underline{x} \mid \underline{r}>$, the command

$$
\mathrm{A}:=\operatorname{FoxMatrix}(\mathrm{G}) \text {; }
$$

constructs the integer matrix $A$ representing the homomorphism $\Delta: \oplus_{\underline{r}} \mathbf{Z} G \rightarrow \oplus_{\underline{x}} \mathbf{Z} G$.

\section{IdentityModuleZBasis(G)}

Given a finitely presented finite group $G=<\underline{x} \mid \underline{r}>$, the command

$$
\mathrm{S}:=\text { IdentityModulezBasis(G); }
$$

constructs a set $S$ of vectors that form a basis for the free abelian group underlying $\pi=$ $\pi_{2} K(\underline{x}, \underline{r})$. Thus the vectors in $S$ have length equal to $|G| \times|\underline{r}|$; the implicit ordering on the elements of $G$ is that given by the standard GAP command

$$
\text { Elements(G); }
$$

for listing the elements of $G$. The set $S$ is consructed by applying the GAP command LLLReducedBasis to an integer matrix with $|\underline{r}||G|$ rows and $|\underline{x}||G|$ columns.

\section{VectorPermutations $(\mathbf{G}, \mathbf{v})$}

Given a finitely presented finite group $G=<\underline{x} \mid \underline{r}>$ and an integer vector $v$ whose length $|v|$ is an integer multiple of $n=|G|$, the command

$$
\mathrm{u}:=\text { VectorPermutations }(\mathrm{G}, \mathrm{v}) \text {; }
$$

constructs a sequence $u=\left\{u_{1}, \ldots, u_{n}\right\}$ of integer vectors $u_{i}$ with each $u_{i}$ obtained by permuting the coordinates of $v$ as follows. The vector $v$ is identified in the usual way with an element in the free module $\mathbf{Z} G \oplus \ldots \oplus \mathbf{Z} G$ (the direct sum of $|v| /|G|$ copies of $\mathbf{Z} G$ ). The vector $u_{i}$ represents the element $g_{i} . v$ in this free module, where $g_{i}$ is the $i$ th element of $G$.

\section{MinimalModuleGenerators(G,S)}

Given a finitely presented finite group $G=<\underline{x} \mid \underline{r}>$ and a set $S=\left\{v_{1}, \ldots, v_{n}\right\}$ of integer vectors of equal length $k=\left|v_{i}\right|$ with $k$ an integer multiple of $|G|$, the command

$$
\mathrm{T}:=\text { MinimalModuleGenerators }(\mathrm{G}, \mathrm{S}) \text {; }
$$

constructs a subset $T \subset S$ as follows. The vectors $v_{i}$ are identified with elements in the free module $\mathbf{Z} G \oplus \ldots \oplus \mathbf{Z} G(k /|G|$ copies of $\mathbf{Z} G)$. Let $<S>\mathbf{Z}$ denote the abelian group consisting of all finite integer linear combinations of the elements in $S$. Let $<T>\mathbf{Z} G$ 
denote the $\mathbf{Z} G$-module generated by the elements of $T$. Then $T$ is constructed to have the property $<S>_{\mathbf{Z}} \subset<T>_{\mathbf{Z} G}$; moreover, no subset of $T$ has this property. This function may use the GAP command LLLReducedBas is several times on an integer matrix with $|\underline{r}||G|$ rows and $|\underline{x}||G|$ columns.

\section{IdentityModuleGenerators $(\mathbf{G})$}

Given a finitely presented finite group $G=<\underline{x} \mid \underline{r}>$, the command

$$
\mathrm{T}:=\text { IdentityModuleGenerators(G); }
$$

constructs a set $T$ of integer vectors $v_{i}$ of length $\left|v_{i}\right|=|G| \times|\underline{r}|$. The set $T$ represents a minimal set of generators for the module of identities $\pi=\pi_{2} K(\underline{x}, \underline{r})$.

\section{IntegralHomology $(\mathbf{G , n})$}

Given a finitely presented finite group $G=<\underline{x} \mid \underline{r}>$ and an integer $n \in\{1,2,3\}$, the command

$$
\mathrm{H}:=\text { IntegralHomology }(\mathrm{G}, \mathrm{n}) \text {; }
$$

constructs the list $H$ of abelian group invariants of the finite homology group $H_{n}(G, \mathbf{Z})$.

\section{Help()}

The command

$$
\operatorname{Help}()
$$

displays on screen the above information about the functions.

The command

$$
\text { Test(); }
$$

checks that the functions are operating correctly on a given computer. Error messages will be produced if the functions produce answers different from those obtained on the authors' computer.

Appendix A. Computational results presented in HTML format

This appendix is available to subscribers to the journal at: http://www.lms.ac.uk/jcm/2/lms99006/appendix-a/.

\section{Appendix B. GAP procedures to compute $\pi$ and $H_{3}(G, \mathbf{Z})$}

This appendix is available to subscribers to the journal at: http://www.lms.ac.uk/jcm/2/lms99006/appendix-b/.

\section{References}

1. Y.-G. BAIK, J. Harlander and S. J. Pride, 'The geometry of group extensions', $J$. Group Theory 1 (1998) 395-416. 94, 105, 105

2. H. J. BAuEs, Combinatorial homotopy and 4-dimensional complexes, de Gruyter Expos. Math. vol. 2 (de Gruyter, Berlin, New York, 1991). 94, 95, 101, 102, 102

3. F. R. BEYL and J. TAPPE, Group extensions, representations, and the Schur multiplier, Lecture Notes in Math. 958 (Springer, 1982). 106, 109 
4. W. A. Bogley and S. J. Pride, 'Calculating generators of $\pi_{2}$ ', Two-dimensional homotopy and combinatorial group theory (ed. C. Hog-Angeloni, W. Metzler and A. J. Sieradski), London Mathematical Society Lecture Note Series 197 (Cambridge University Press, 1993) 157-188. 94, 102, 105, 105

5. W. Bosma, J.CAnnon and C. Playoust, 'The Magma algebra system I: the user language', J. Symbolic Comput. 24 (1997) 235-265. 94, 100

6. R. Brown and P. J. Higgins, 'Tensor products and homotopies for $\omega$-groupoids and crossed complexes', J. Pure Applied Algebra 47 (1987) 1-33. 102

7. R. BRown and J. HuEBSCHMANN, 'Identities among relations', Low dimensional topology (ed. R. Brown and T. L. Thickstun), London Mathematical Society Lecture Note Series 48 (1982) 153-202. 94, 95, 96, 96, 101, 102

8. R. Brown, D. L. Johnson and E. F. Robertson, 'Some computations of nonabelian tensor products of groups', J. Algebra 111 (1987) 177-202 106

9. R. Brown and T. Porter, 'On the Schreier theory of nonabelian extensions: generalisations and computations', Proc. Royal Irish Academy 96A (1996) 213-227. 102

10. R. Brown and A. Razak Salleh, 'Free crossed resolutions of groups and presentations of modules of identities among relations', LMS J. Comput. Math. 2 (1999) 28-61 http://www.lms.ac.uk/jcm/2/lms99001. 94, 99, 99

11. T.-W. J. ChOu and G. E. Collins, 'Algorithms for the solution of linear diophantine equations', SIAM J. Computing 11 (1982) 687-708. 97

12. H. S. M. COXETER and W. O. J. MOSER, Generators and relations for discrete groups, 4th edn (Springer-Verlag, 1979). 94

13. M. Dyer, 'Crossed and precrossed modules', Two-dimensional homotopy and combinatorial group theory (ed. C. Hog-Angeloni, W. Metzler and A. J. Sieradski), London Mathematical Society Lecture Note Series 197 (Cambridge University Press, 1993) 125-156. 101

14. G. ElLIS, 'Nonabelian exterior products of groups and an exact sequence in the homology of groups', Glasgow Math. J. 29 (1987) 13-19. 109

15. G. ElLIS, 'Crossed squares and combinatorial homotopy', Math. Zeitschrift 214 (1993) 93-110. 94

16. G. Ellis, 'On the computation of certain homotopical functors', LMS J. Comput. Math. 1 (1998) 25-41 http://www.lms.ac.uk/jcm/1/lms97004. 112

17. G. ElLIS, 'Enumerating prime-power homotopy k-types', Math. Zeitschrift (to appear). 94, 109

18. G. Ellis and A. McDermott, 'Tensor products of prime-power groups', J. Pure Applied Algebra 132 (1998) 119-128. 109

19. G. Ellis and T. PORTER, 'Free and projective crossed modules and the second homology group of a group', J. Pure Applied Algebra 40 (1986) 27-31. 101, 109

20. D. B. A. EPSTEIN, 'Finite presentations of groups and 3-manifolds', Quart. J. Math. Oxford (2) 12 (1961) 205-212. 106

21. The GAP Group, GAP - Groups, Algorithms and Programming (School of Mathematical and Computational Sciences, University of St. Andrews, Scotland, 1998). 94, $97,111,114$ 
22. J. R. J. Groves, 'An algorithm for computing homology groups', J. Algebra 194 (1997) 331-361. 94, 94, 94, 100, 100, 100

23. J. HARLANDER, 'Closing the relation gap by direct product stabilization', J. Algebra 182 (1996) 511-521. 106, 108, 110

24. L. G. KovÁcs, 'Finite groups with trivial multiplicator and large deficiency', GroupsKorea '94 (Pusan) (de Gruyter, Berlin, 1995) 211-225. 106

25. L. Johansson, L. LAmbe and E. SköldBerg, 'Normal forms and iterative methods for constructing resolutions', Preprint, Stockholm, 1998. 94

26. D. L. Johnson, Topics in the theory of group presentations, London Mathematical Society Lecture Note Series 42 (Cambridge University Press, 1980). 104, 105, 110

27. A. K. Lenstra, H. W. Lenstra and L. Lovasz, 'Factoring polynomials with rational coefficients', Math. Annalen 261 (1982) 515-534. 94, 97, 97, 111

28. J. G. Ratcliffe, 'Free and projective crossed modules', J. London Math. Soc. 22 (1980) 66-74. 101

29. J. J. Rotman, An introduction to algebraic topology, Graduate Texts in Math. 119 (Springer-Verlag, 1988). 108, 111

30. A. J. SiERADSKI, 'Algebraic topology for two-dimensional complexes', Twodimensional homotopy and combinatorial group theory (ed. C. Hog-Angeloni, W. Metzler and A. J. Sieradski), London Mathematical Society Lecture Note Series 197 (Cambridge University Press, 1993) 51-96. 95, 96, 96, 101, 102

31. R. G. Swan, 'Minimal resolutions for finite groups', Topology 4 (1965) 193-208. 106, $108,109,110,110$

32. J. H. C. Whitehead, 'Combinatorial homotopy II', Bull. American Math. Soc. 55 (1949) 453-496. 95

Graham Ellis graham.ellis@nuigalway.ie http://hamilton.nuigalway.ie

Max-Planck-Institut für Mathematik

D-53225 Bonn

and

Mathematics Department

National University of Ireland

Galway

Irina Kholodna irina.kholodna@nuigalway.ie

Mathematics Department

National University of Ireland

Galway 